The Astrophysical Journal, 644:510-524, 2006 June 10

(C) 2006. The American Astronomical Society. All rights reserved. Printed in U.S.A.

\title{
THE ORBIT AND OCCULTATIONS OF KH 15D
}

\author{
Joshua N. Winn, ${ }^{1,2,3}$ Catrina M. Hamilton, ${ }^{4,5}{ }^{\text {William J. Herbst, }}{ }^{6}$ Jennifer L. Hoffman, $^{7}$ \\ Matthew J. Holman, ${ }^{1}$ John A. Johnson, ${ }^{7}$ and Marc J. Kuchner ${ }^{8}$ \\ Received 2005 December 30; accepted 2006 February 15
}

\begin{abstract}
The unusual flux variations of the pre-main-sequence binary star KH 15D have been attributed to occultations by a circumbinary disk. We test whether or not this theory is compatible with newly available data, including recent radial velocity measurements, CCD photometry over the past decade, and photographic photometry over the past 50 years. We find the model to be successful, after two refinements: a more realistic motion of the occulting feature and a halo around each star that probably represents scattering by the disk. The occulting feature is exceptionally sharp edged, raising the possibility that the dust in the disk has settled into a thin layer and providing a tool for fine-scale mapping of the immediate environment of a T Tauri star. However, the window of opportunity is closing, as the currently visible star may be hidden at all orbital phases by as early as 2008 .
\end{abstract}

Subject headings: circumstellar matter — open clusters and associations: individual (NGC 2264) stars: individual (KH 15D) - stars: pre-main-sequence

\section{INTRODUCTION}

After a newborn low-mass star emerges from the dust of its parent molecular cloud, it spends a few million years as a T Tauri star. This phase is characterized by optical variability and chromospheric emission lines, as the star accretes gas, propels a bipolar outflow, and contracts onto the main sequence (see, e.g., the review by Bertout 1989). A conventional boundary line is drawn between classical and weak-lined $\mathrm{T}$ Tauri stars, depending on whether the equivalent width of $\mathrm{H} \alpha$ emission is greater or smaller than some threshold value, typically 5-10 $\AA$. Of course, this boundary line is somewhat arbitrary, and transitional cases are to be expected. But we are aware of only one T Tauri star, KH 15D, that seems to mock this definition by alternating between classical and weak-lined with clocklike regularity (Hamilton et al. 2003).

This is not the only unusual property of KH 15D. Although it was classified as a variable member of the young cluster NGC 2264 by Badalian \& Erastova (1970), the peculiarity of its variations was not appreciated until Kearns \& Herbst (1998) found that the object fades by 3 mag every 48 days. Hamilton et al. (2001) and Herbst et al. (2002) proposed that the star was being periodically eclipsed by circumstellar material. Further monitoring revealed qualitative changes in the eclipses, most notably a gradual increase in their depth and duration, as well as the progressive disappearance of a "rebrightening" event that once characterized the mideclipse phase. The dramatic growth in the equivalent width of $\mathrm{H} \alpha$ emission occurs during the transition from the bright state to the faint state. Apparently, the occulting material blocks the photosphere of the star and thereby reduces

\footnotetext{
${ }^{1}$ Harvard-Smithsonian Center for Astrophysics, Mail Stop 51, 60 Garden Street, Cambridge, MA 02138.

${ }^{2}$ Hubble Fellow.

${ }^{3}$ Current address: Department of Physics, Massachusetts Institute of Technology, 77 Massachusetts Avenue, Cambridge, MA 02139.

4 Astronomy Department, Mount Holyoke College, 50 College Street, South Hadley, MA 01075.

5 Five College Astronomy Department, University of Massachusetts, Amherst, MA 01003.

6 Van Vleck Observatory, Wesleyan University, Middletown, CT 06459.

7 Department of Astronomy, Mail Code 3411, University of California, Berkeley, CA 94720.

${ }^{8}$ NASA Goddard Space Flight Center, Greenbelt, MD 20771.
}

the contrast between the photosphere and the $\mathrm{H} \alpha$-emitting region: the star has a "natural coronagraph" (Hamilton et al. 2003). Eclipse-related variations in both optical polarization (Agol et al. 2004) and molecular $\mathrm{H}_{2}$ emission (Deming et al. 2004) have also been observed.

For several years the cause of the eclipses and of their evolution were the subject of much speculation (Grinin \& Tambovtseva 2002; Herbst et al. 2002; Winn et al. 2003; Barge \& Viton 2003; Klahr \& Bodenheimer 2003; Agol et al. 2004; Barrado y Navascués et al. 2004). Recently, it was proposed that KH $15 \mathrm{D}$ is an eccentric binary system that is being occulted by the edge of a circumbinary disk (Winn et al. 2004; Chiang \& Murray-Clay 2004). In this scenario, we are viewing the system nearly edge-on, and the sky projection of the disk acts as a dark screen that gradually covers the binary. The advance of the screen is a consequence of the nodal precession of the disk, which is inclined relative to the binary plane. At present, one member of the binary is hidden at all orbital phases, and the other member undergoes an eclipse each time its orbital motion brings it behind the screen. Winn et al. (2004; hereafter, Paper I) showed that this model unified the diverse properties of $\mathrm{KH} 15 \mathrm{D}$ and provided a quantitative fit to the available measurements of the eclipses and their evolution. Chiang \& Murray-Clay (2004) arrived at the same idea and argued that the disk must be warped and radially narrow (a "ring") in order to maintain rigid precession.

It has since been confirmed that KH $15 \mathrm{D}$ is a spectroscopic binary with an eccentric orbit (Johnson et al. 2004). In addition, a wealth of new photometric data have become available, from analyses of archival photographs (Johnson et al. 2005; Maffei et al. 2005) and modern CCD observations (Hamilton et al. 2005; Barsunova et al. 2005; Kusakabe et al. 2005). Our main motivation for the work described in this paper was to test whether or not the circumbinary-disk model is consistent with these new data. In $\S \S 2$ and 3 we describe our compilation of the data, and in $\S 4$ we specify the model and its parameters. Section 5 is a direct extension of Paper I: we attempt to fit the observed times and durations of the eclipses and the observed radial velocity variations.

A second motivation for this work was to understand certain features of the data that had not previously been explained. What is the origin of the light that is received during eclipses? Why is the depth of the eclipses slowly increasing with time? What 
causes the nearly repeatable flux variations that are seen throughout ingress, mideclipse, and egress? We found that by making a minor elaboration to the model-surrounding each of the stars with a faint blue halo-all of these observations could be accurately described. This is shown in $\S 6$.

Finally, in $\S 7$ we discuss the interpretation of the fitted model parameters, in particular those that describe the halos. The most likely interpretation is that scattering by the disk creates an apparent halo around each star. We suggest some future observations and calculations that may clarify the interpretation. If these are successful, and if the occulting edge is as sharp as we suspect, then it will be possible to use the occulting edge to map out the environment of the visible star with wondrously fine detail. This would broaden the significance of this system from being a fascinating puzzle to being a crucial fortuitous case that will provide a deeper understanding of all young stars.

\section{PHOTOMETRY}

Two types of photometry are available for this system: photographic photometry, generally from years before 1985, and CCD photometry, from 1995 to the present. In all cases the measurements refer to the total light from the system, because the system has not been spatially resolved (with the possible exception of an $\mathrm{H}_{2}$ filament observed by Tokunaga et al. 2004). The data are very heterogeneous, having been gathered with dozens of different telescopes and reduced with different procedures. This section describes the available data and our efforts to allow them to be modeled within a single framework. In general, our attitude was cautious, in the sense that we wished to maximize the reliability of the data even at the expense of discarding some of the data.

\subsection{Photographic Photometry}

As a fairly unobscured example of a young stellar cluster, rich with variable stars, NGC 2264 has long been a popular target for photometric campaigns. Many photographic plates of NGC 2264 taken over the past century have been preserved in observatory archives. Four analyses of archival observations of KH 15D have been published:

1. Winn et al. (2003) used blue-sensitive exposures from the Harvard College Observatory to show that in the first half of the twentieth century, the system was rarely (if ever) fainter than its modern out-of-eclipse level by more than 1 mag. Given the number of plates analyzed, they concluded that the system spent less than $20 \%$ of the time in such a faint state. More accurate photometry was impossible because of the glare from a nearby star, HD 47887.

2. Johnson \& Winn (2004) performed profile photometry on a digitized set of infrared-sensitive exposures from the $92 / 67 \mathrm{~cm}$ Schmidt telescope of the Astrophysical Observatory of Asiago, Italy, from the time period 1967-1982. The glare from HD 47887 is less problematic on the infrared plates than on the blue plates. The relative magnitudes have a typical accuracy of $0.1 \mathrm{mag}$. They were placed on the standard Cousins $I$-band magnitude scale using a set of reference stars whose $I$-band magnitudes had been measured with CCD observations by Flaccomio et al. (1999). The zero-point uncertainty is $0.14 \mathrm{mag}$.

3. Maffei et al. (2005) analyzed a collection of blue and infrared plates that were originally obtained by the lead author, P. Maffei, between 1955 and 1970 using five different telescopes. Relative photometry was performed visually, with an estimated accuracy ranging from 0.05 to 0.2 mag depending on the telescope. The blue magnitudes were converted to the Johnson $B$-band using

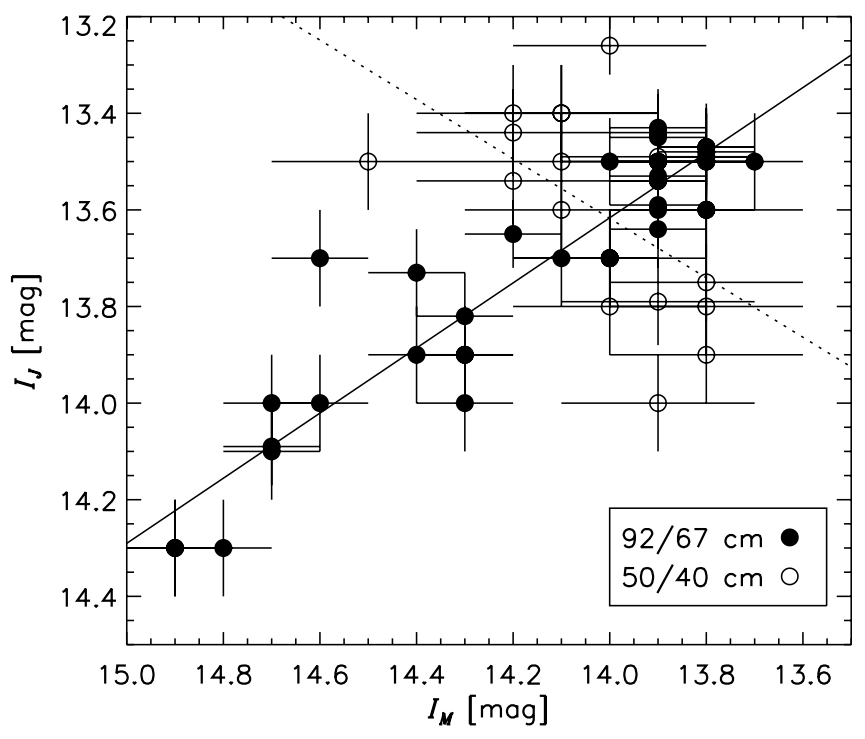

FIG. 1.-Comparison between the $I$-band magnitudes of KH 15D reported by two different groups based on the same photographic plates. Each point represents a single infrared plate, with $I_{\mathrm{M}}$ defined as the value reported by Maffei et al. (2005), and $I_{\mathrm{J}}$ as the value reported by either Johnson \& Winn (2004) or Johnson et al. (2005). Different symbols correspond to different telescopes. The solid line is a linear least-squares fit to the $92 / 67 \mathrm{~cm}$ data, and the dotted line is a linear least-squares fit to the $50 / 40 \mathrm{~cm}$ data.

a set of reference stars with photographic and photoelectric $B$ magnitudes given by Walker (1956). The infrared magnitudes were converted to the Cousins $I$ band, after adopting an $I$-band magnitude for each reference star based on an extrapolation of its $B-V$ color.

4. Johnson et al. (2005) gathered and digitized a collection of 87 plates, representing 8 different telescopes and 22 different filter/ emulsion combinations, from the time period 1954-1997. The magnitudes of $\mathrm{KH} 15 \mathrm{D}$ were measured and reduced to UBVRI magnitudes using the procedure of Johnson \& Winn (2004).

The latter 3 samples have 56 plates in common, giving us the opportunity to check for consistency between the different photometric methods. The plates in common are all infrared plates from one of the two Asiago Schmidt telescopes: 38 are from the $92 / 67 \mathrm{~cm}$ telescope, and 18 are from the $50 / 40 \mathrm{~cm}$ telescope. Figure 1 shows a comparison of $I_{\mathrm{M}}$, the magnitude reported by Maffei et al. (2005), and $I_{\mathrm{J}}$, the magnitude reported by either Johnson \& Winn (2004) or Johnson et al. (2005) based on the same plate. There are significant discrepancies. For the $92 / 67 \mathrm{~cm}$ plates, the relation between $I_{\mathrm{J}}$ and $I_{\mathrm{M}}$ is well fitted by a straight line,

$$
I_{\mathrm{J}}-13.80=0.67\left(I_{\mathrm{M}}-14.27\right)
$$

as plotted in Figure 1. This relation may reflect the different methods for determining the zero point and for coping with the nonlinear response of the emulsions. For the $50 / 40 \mathrm{~cm}$ plates, there is no obvious relation between $I_{\mathrm{J}}$ and $I_{\mathrm{M}}$. The best-fitting line (Fig. 1, dotted line) represents an anti-correlation between the two sets of results, which does not make sense, suggesting that the photometric uncertainties have been underestimated by one or both sets of authors. Of all the infrared plates, Johnson et al. (2005) found the $50 / 40 \mathrm{~cm}$ plates to be the most difficult to analyze, because of the large plate scale and consequently poor sampling of the stellar images.

In this work we attempt to fit only the $I$-band data, mainly because the majority of modern CCD observations were made in 


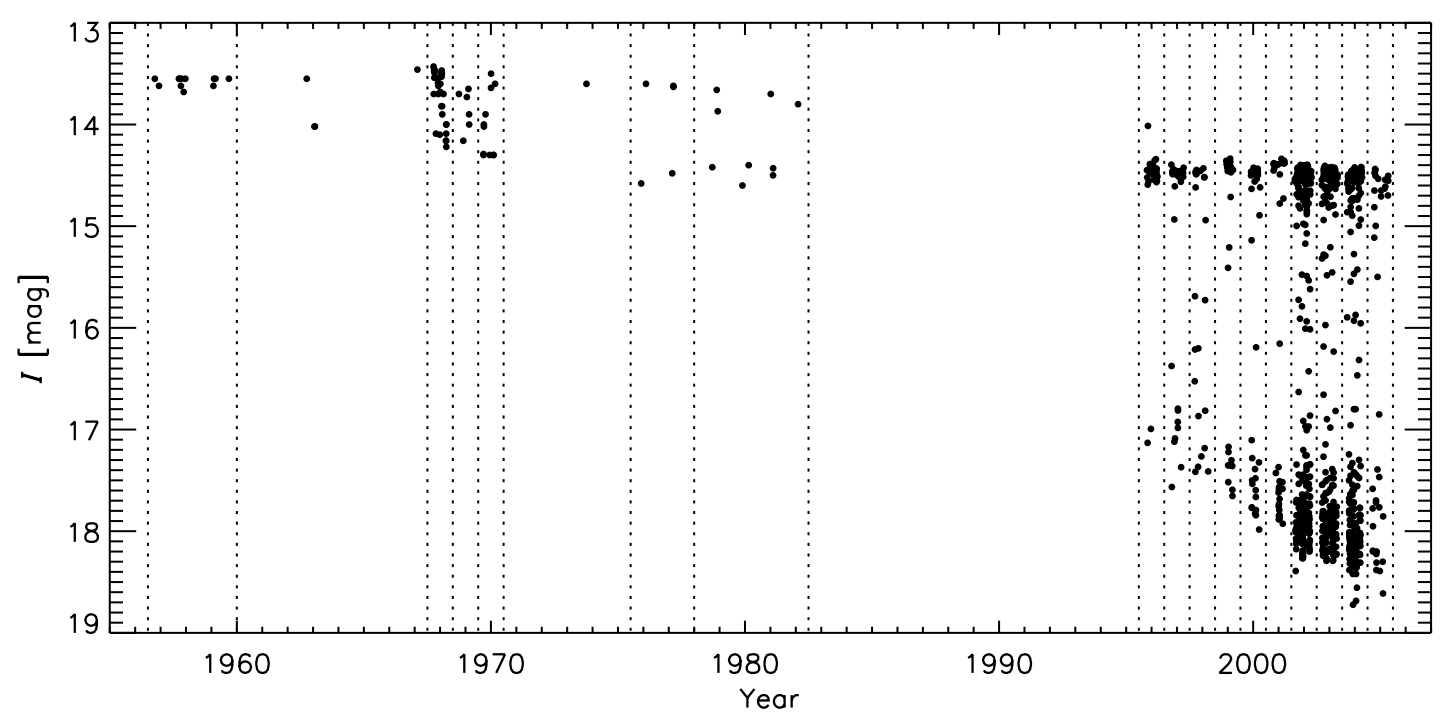

Fig. 2.-Fifty years of Cousins $I$-band photometry of KH 15D. The post-1995 data have been averaged into $6 \mathrm{hr}$ bins. (The dotted lines mark the time ranges that are plotted in the panels of Fig. 12.)

the $I$ band and also because we judge the photographic photometry to be most reliable for the infrared plates. We briefly discuss the data at other wavelengths in $\S 7$, but we do not use those data to determine our model parameters. Because we do not know how to resolve the discrepancy between the two different sets of $I$-band results, we do not consider the Asiago 50/40 cm data further. For the Asiago 92/67 cm data, we use $I_{\mathrm{J}}$ when it is available, and otherwise we place $I_{\mathrm{M}}$ onto the $I_{\mathrm{J}}$ system ${ }^{9}$ using equation (1). For the data from all of the other telescopes, we use the published results without modification. The resulting data set consists of 81 measurements. The data are plotted in Figure 2, along with the CCD photometry from more recent times.

\subsection{CCD Photometry}

Over the last decade, $\mathrm{KH} 15 \mathrm{D}$ has been monitored intensively. The first few years of observations used the $0.6 \mathrm{~m}$ telescope at Van Vleck Observatory on the campus of Wesleyan University, in Connecticut, and were designed to monitor the variability of a large sample of stars in NGC 2264. After two seasons of observing, Kearns \& Herbst (1998) identified KH 15D as an especially interesting variable star. Hamilton et al. (2001) focused specifically on KH $15 \mathrm{D}$, presenting a third year of photometry, along with optical spectroscopy and a discussion of possible explanations for the peculiar eclipses. An observing campaign involving many observatories was organized, and the first year's results were described by Herbst et al. (2002). Most recently, Hamilton et al. (2005) presented both new photometry and a comprehensive summary of $9 \mathrm{yr}$ of CCD photometry involving a dozen different telescopes. We refer the reader to that work for a detailed discussion of the calibration and internal consistency of the various data sets involved. Most of the photometry is in the Cousins I band. A notable exception is the season of 2001-2002, when simultaneous Johnson VI measurements were made with the Yale $1 \mathrm{~m}$ telescope on nearly every clear night. There are also a smaller number of other observations in the $U B V R$ bands.

Barsunova et al. (2005) have independently monitored KH 15D in the Johnson $V$ and Cousins $R$ and $I$ bands since 2002, using the $0.7 \mathrm{~m}$ telescope of the Crimean Astrophysical Observatory.

\footnotetext{
${ }^{9}$ We transformed $I_{\mathrm{M}}$ into $I_{\mathrm{J}}$, rather than the other way around, because the zero point of the $I_{\mathrm{J}}$ scale was set with reference to CCD $I$-band observations, and we therefore expected $I_{\mathrm{J}}$ to provide better consistency with the modern photometry.
}

Eighteen of their $I$-band measurements were taken within $6 \mathrm{hr}$ of a measurement by Hamilton et al. (2005), which, as in the previous section, gives us an opportunity to check for consistency between the teams' different photometric procedures. We found them to be consistent with the linear transformation

$$
I_{\mathrm{H}}=0.97 I_{\mathrm{B}}+0.27,
$$

which is essentially a difference in the zero point of the magnitude scale.

Recently, Kusakabe et al. (2005) presented near-infrared $\left(J H K_{s}\right)$ photometry of KH 15D spanning two observing seasons, from 2003 to 2005. As stated previously, in what follows we are concerned almost entirely with the $I$-band data, although we do remark on the near-infrared data in $\S 7$.

For our model-fitting purposes, we considered all of the $I$-band data of Hamilton et al. (2005) and Barsunova et al. (2005), after transforming the latter data set according to equation (2) for the sake of uniformity. In addition, V. Grinin (2005, private communication) kindly provided us the results from the 20042005 observing season that have not yet been published, which we also transformed according to equation (2) and added to the data set under consideration. In total, there are 6780 data points, of which 87 are from the Russian group. The uncertainties range widely from a few millimagnitudes, for some observations when $\mathrm{KH} 15 \mathrm{D}$ was in its bright state, to $0.25 \mathrm{mag}$, when it was in its faint state.

\subsection{Summary of the Photometry}

The entire $I$-band time series, from 1956 until the present, is shown in Figure 2. Many interesting features of the system's photometric history can be seen in this figure. Before about 1960, there is not much evidence for variability greater than $0.1 \mathrm{mag} .{ }^{10}$

\footnotetext{
10 Maffei et al. (2005) found evidence for periodic variations in $B$ between 1958 and 1963, a time when $I$-band variations at the same level could be excluded. Most of the relevant data is from the Asiago 50/40 cm Schmidt telescope. In $\S 2.1$ we showed that two different groups arrived at significantly different results when analyzing the same infrared plates from that telescope, suggesting that the uncertainties may be larger than they appear. The uncertainties should be still larger for the blue plates because of the difficulty presented by the neighboring bright star. Hence, although the possibility that the eclipses began in $B$ prior to $I$ is interesting and worthy of further investigation, we do not consider this possibility further in this paper.
} 

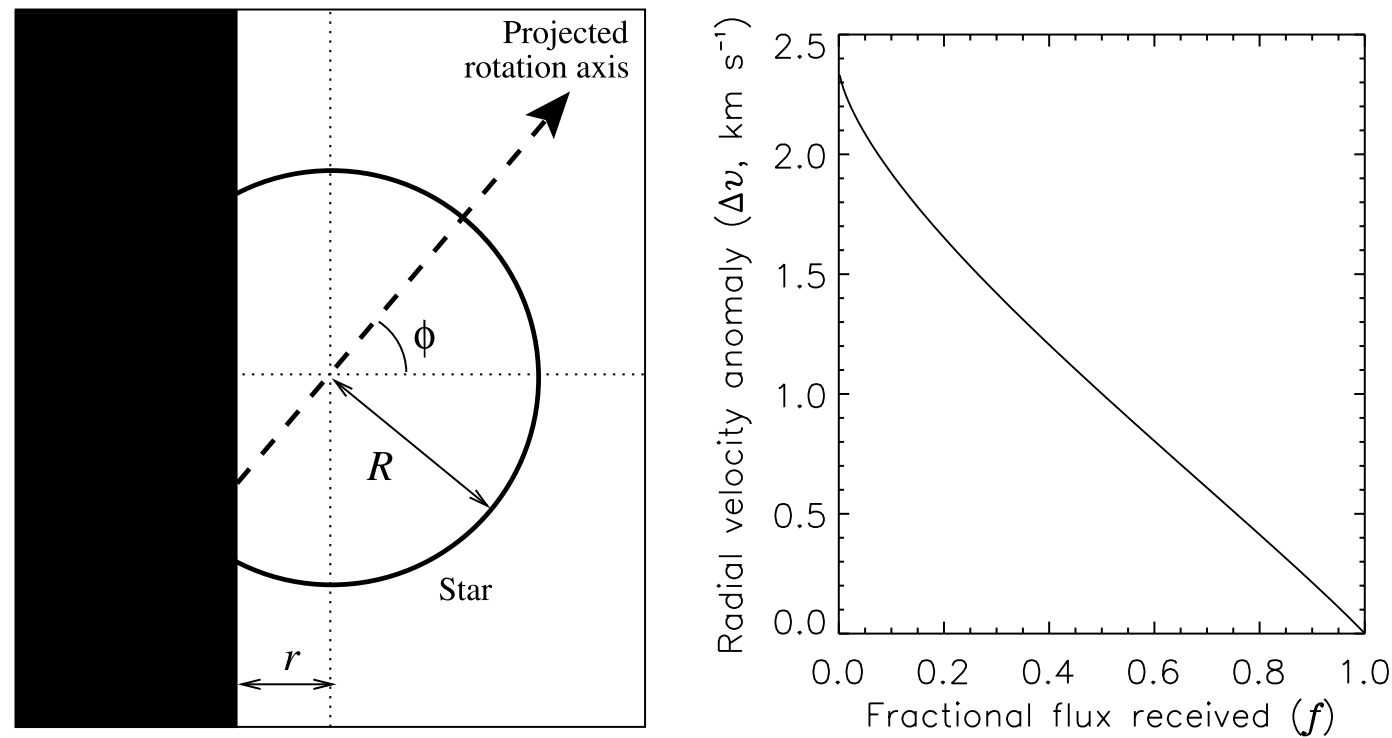

FIG. 3.- Rossiter-McLaughlin effect produced by a straight-edged, semi-infinite occulter. Left: Diagram of the star, its projected rotation axis, and the occulting edge. Right: Magnitude of the Rossiter-McLaughlin effect, using eqs. (3) and (4) and assuming $V_{\text {rot }} \sin I_{\star}=6.9 \mathrm{~km} \mathrm{~s}^{-1}$ and $\phi=20^{\circ}$, as estimated for KH $15 \mathrm{D}$.

Beginning in the early 1960s and lasting until at least the early 1980 s, the system varied between $I \approx 13.6$ and 14.5 . By 1995 the variations were between 14.5 and a faint state that has gradually darkened from about 17 to 18.5. Although it is not apparent in Figure 2, both the pre-1980 and post-1995 variations are periodic, with a 48.4 day period, and the phase of minimum light shifted by nearly $180^{\circ}$ between those time periods (Johnson \& Winn 2004).

\section{RADIAL VELOCITIES}

Possible time variations of the Doppler shift of starlight from KH 15D were first reported by Hamilton et al. (2001, 2003). Subsequently, Johnson et al. (2004) showed that KH 15D is indeed a spectroscopic binary, based on a series of high-resolution optical spectra using telescopes at the Keck, Magellan, and McDonald observatories. With 16 measurements spanning $1.3 \mathrm{yr}$ with accuracies ranging from 0.2 to $0.6 \mathrm{~km} \mathrm{~s}^{-1}$, Johnson et al. (2004) showed that the Doppler shifts could be explained as radial velocity variations of a star in an eccentric Keplerian orbit.

If the occulting edge of $\mathrm{KH} 15 \mathrm{D}$ is sharp enough to resolve the stellar photosphere, then the radial velocity measurements taken during ingress or egress are subject to systematic errors due to the Rossiter-McLaughlin effect (Rossiter 1924; McLaughlin 1924). This term refers to the apparent velocity perturbation of a partially occulted star due to stellar rotation. For example, if only the receding half of a star is visible, then the starlight is redshifted in excess of the shift one would expect from only the star's centerof-mass motion. This effect has long been observed during eclipses of binary stars (see, e.g., Worek 1996; Rauch \& Werner 2003) and more recently during transits of extrasolar planets (Bundy \& Marcy 2000; Queloz et al. 2000; Snellen 2004; Winn et al. 2005). To estimate the magnitude of the effect, suppose that a star of radius $R$ is occulted by a straight-edged semi-infinite screen, as depicted in Figure 3 (left). The minimum distance between the edge and the stellar center is $r$. The angle between the normal to the edge and the sky projection of the stellar north pole is $\phi$. The fraction of the total stellar flux $(f)$ and the mean line-of-sight velocity $(\Delta V)$ of the exposed portion of the star are given by

$$
\begin{aligned}
f & =\frac{1}{\pi}\left(\cos ^{-1} u-u \sqrt{1-u^{2}}\right), \\
\Delta V & =\frac{2}{3} V_{\text {rot }} \sin I_{\star} \sin \phi\left[\frac{\left(1-u^{2}\right)^{3 / 2}}{\cos ^{-1} u-u \sqrt{1-u^{2}}}\right],
\end{aligned}
$$

where $u=r / R, V_{\text {rot }}$ is the stellar rotation speed, and $I_{\star}$ is the inclination of the stellar rotation axis with respect to the line of sight. These expressions neglect differential rotation and limb darkening.

For the case of KH 15D, Hamilton et al. (2005) measured $V_{\text {rot }} \sin I_{\star}=6.9 \pm 0.3 \mathrm{~km} \mathrm{~s}^{-1}$ for the currently visible star. We estimate $\phi \approx 20^{\circ}$, using the model of Paper I and assuming the stellar rotation axis to be perpendicular to the stellar orbital plane. Figure 3 (right) shows the resulting magnitude of $\Delta V$ as a function of $f$. For the perturbations to be smaller than $0.2 \mathrm{~km} \mathrm{~s}^{-1}$, and hence smaller than the measurement uncertainties in the radial velocity data, we need $f>0.9$. Thus, for model-fitting purposes, we consider only the 12 radial velocity measurements that were obtained when the system flux was $90 \%$ or greater of its mean outof-eclipse flux. This is a cautious procedure, in the sense that the systematic error is smaller for the more realistic case of a star with limb darkening and an occulting edge that is not perfectly sharp. ${ }^{11}$

We made two other minor adjustments to the data published by Johnson et al. (2004). First, we corrected the time stamps on some of the Keck measurements, which were in error by 0.5 day because of a confusion between Julian and Modified Julian dates. Second, we added $0.25 \mathrm{~km} \mathrm{~s}^{-1}$ in quadrature to the uncertainties of the data labeled " $b$ " in Table 1 of Johnson et al. (2004). In those cases the radial velocity scale was calibrated using only

11 We also tried using all the data with $f>0$, after enlarging the error bars to encompass the expected systematic errors. In that case, the procedure described in $\S 4$ resulted in significantly poorer fits, but the optimized values of the parameters were similar to those reported in Table 1. 
TABLE 1

Radial Velocity Measurements of KH 15D

\begin{tabular}{|c|c|c|}
\hline Julian Date & Relative Flux $(f)$ & $v_{r}$ \\
\hline $2,452,242.7649 \ldots$ & 0.0 & $9.00 \pm 0.32$ \\
\hline 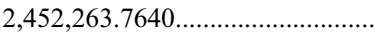 & 0.0 & $12.30 \pm 0.60$ \\
\hline $2,452,573.0749 \ldots$ & 1.0 & $1.70 \pm 0.20$ \\
\hline $2,452,576.0111 \ldots \ldots \ldots \ldots \ldots \ldots \ldots \ldots \ldots$ & 1.0 & $3.00 \pm 0.30$ \\
\hline $2,452,624.7388 \ldots$. & 1.0 & $3.10 \pm 0.32$ \\
\hline $2,452,653.9689 \ldots \ldots \ldots \ldots \ldots \ldots \ldots \ldots \ldots$ & 0.6 & $3.30 \pm 0.20$ \\
\hline $2,452,678.9007 \ldots \ldots$ & 0.7 & $9.00 \pm 0.56$ \\
\hline 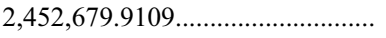 & 0.2 & $11.50 \pm 0.56$ \\
\hline $2,452,707.5117 \ldots$. & 1.0 & $0.80 \pm 0.32$ \\
\hline $2,452,947.0845 \ldots \ldots \ldots \ldots \ldots \ldots \ldots \ldots \ldots$ & 0.9 & $1.20 \pm 0.47$ \\
\hline $2,452,948.0948 \ldots \ldots \ldots \ldots \ldots \ldots \ldots \ldots \ldots$ & 0.9 & $1.80 \pm 0.39$ \\
\hline $2,453,008.7620 \ldots \ldots$. & 1.0 & $1.40 \pm 0.65$ \\
\hline 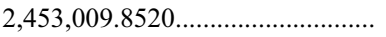 & 1.0 & $1.70 \pm 0.47$ \\
\hline $2,453,014.7600 \ldots \ldots \ldots \ldots \ldots \ldots \ldots \ldots \ldots \ldots \ldots \ldots \ldots \ldots \ldots . \ldots \ldots$ & 0.9 & $5.80 \pm 0.47$ \\
\hline 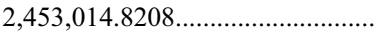 & 0.9 & $5.40 \pm 0.30$ \\
\hline 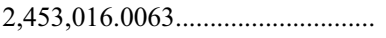 & 0.4 & $7.00 \pm 0.32$ \\
\hline $2,453,044.8341 \ldots \ldots \ldots \ldots \ldots \ldots \ldots \ldots \ldots$ & 1.0 & $1.80 \pm 0.20$ \\
\hline $2,453,045.8282 \ldots \ldots \ldots \ldots \ldots \ldots \ldots \ldots \ldots \ldots \ldots \ldots \ldots \ldots \ldots . \ldots . .$. & 1.0 & $1.25 \pm 0.20$ \\
\hline
\end{tabular}

Notes.-From Hamilton et al. (2003) and Johnson et al. (2004), modified as described in $\S 3$. Positive velocity corresponds to motion away from the Sun.

one reference star and the quoted error estimate was statistical, i.e., based only on the noise in the spectrum of KH 15D. In the other cases (labeled "a") multiple reference stars were available, and it was found that the statistical error was smaller than the variance in the results of using different choices for the reference star. Using the "a" results we estimated that the systematic error in the calibration is $\approx 0.25 \mathrm{~km} \mathrm{~s}^{-1}$. For convenience, the complete and updated list of radial velocity measurements is given in Table 1. Only the 12 measurements with $f \geq 0.9$ were used in the model-fitting procedure.

\section{THE MODEL OF KH 15D}

\subsection{Definition of Parameters}

Our model consists of two stars (A and B) in a Keplerian orbit and a straight-edged semi-infinite occulting screen representing the sky projection of a circumbinary disk. Star A is the star that is currently undergoing periodic eclipses and for which radial velocity variations have been measured. The stars have masses denoted by $M_{\mathrm{A}}$ and $M_{\mathrm{B}}$, and radii $R_{\mathrm{A}}$ and $R_{\mathrm{B}}$. The orbit is specified by the period $(P)$, eccentricity $(e)$, argument of pericenter $(\omega)$, inclination with respect to the sky plane $(I)$, heliocentric radial velocity of the center of mass $(\gamma)$, and a particular time of pericenter passage $\left(T_{p}\right)$.

A diagram of the coordinate system ${ }^{12}$ is given in Figure 4. The $X$-Y plane is the sky plane. The $X$-axis is chosen to be along the line of nodes, with the ascending node of star B at $X>0$. The location and orientation of the occulting screen are specified by its point of intersection between the edge and the $Y$-axis $\left(Y_{E}\right)$ and by the angle that the edge makes with the $X$-axis $\left(\theta_{E}\right)$. In Paper I we assumed that the screen moves uniformly and without rotation, i.e., $Y_{E}$ was taken to be a linear function of time, and $\theta_{E}$ was taken to be a constant. In $\oint 5$ we consider these same assumptions, but we also consider more general cases that we argue are more realistic.

Once the orbital parameters and the screen's trajectory are specified, five "orbital contact times" can be defined (see Fig. 5).

\footnotetext{
12 Although our model is the same as that presented previously in Paper I, we have chosen a different coordinate system for convenience.
}

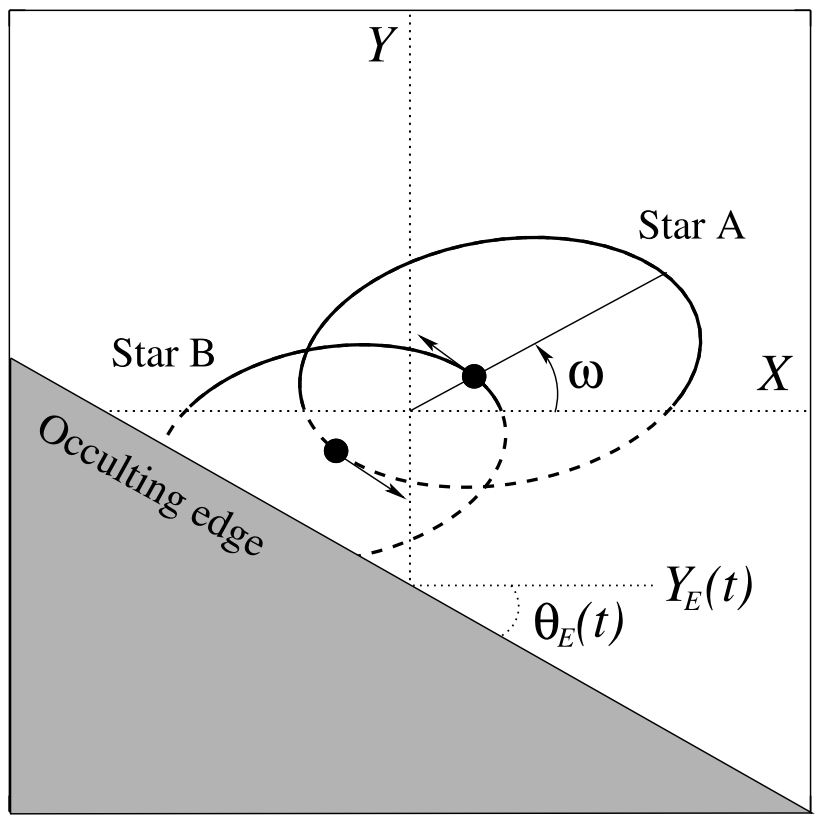

FIG. 4.- Coordinate system. The $X-Y$ plane is the sky plane and the $Z$-axis points toward the observer. The orbital plane is inclined by an angle $I$ with respect to the sky plane. The line of nodes is the $X$-axis, with the ascending node of star B occurring at $X>0$. The $Z>0$ portions of the orbit are marked with solid lines, and the $Z<0$ portions are marked with dashed lines. The stars are shown at pericenter, and $\omega$ is the argument of pericenter. The instantaneous location of the occulting edge is specified by $Y_{E}$, its intersection with the $Y$-axis, and by $\theta_{E}$, the angle that the edge makes with the $X$-axis. A configuration with $I<90^{\circ}, Y_{E}<0$, and $\theta_{E}<0$ is shown.

First contact $\left(t_{1}\right)$ occurs when the occulting edge is tangent to the sky-projected orbit of star B for the first time. Before first contact, no eclipses occur. Figure 2 suggests that first contact occurred between 1959 and 1967. Second contact $\left(t_{2}\right)$ occurs when the occulting edge is first tangent to the sky-projected orbit of star A. Between $t_{1}$ and $t_{2}$, star $\mathrm{B}$ is periodically occulted, but we receive

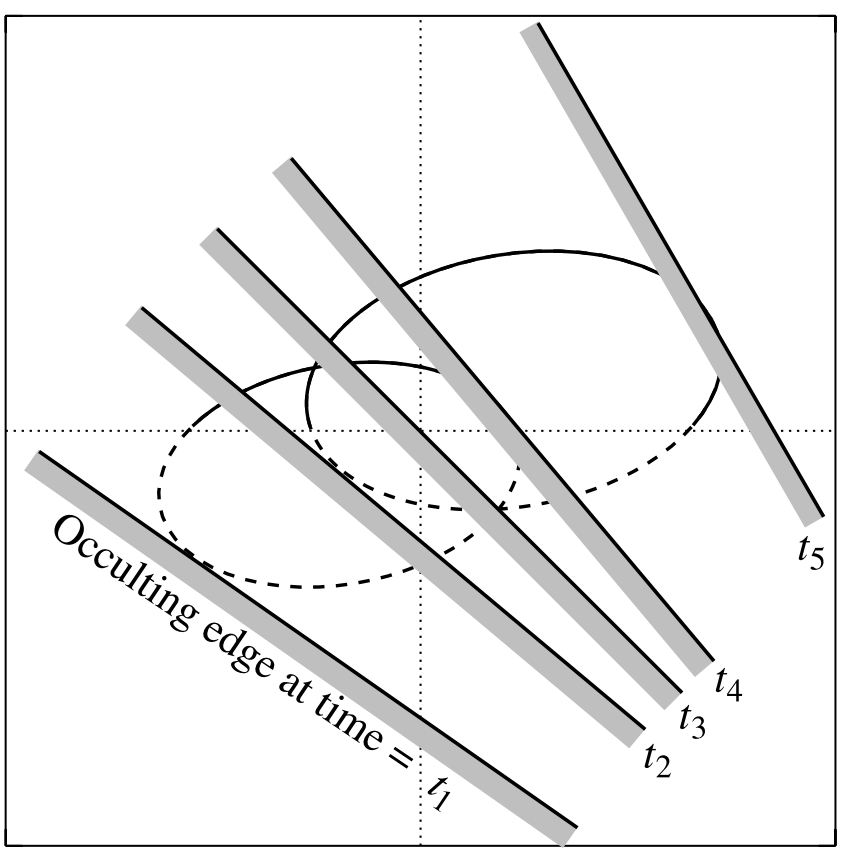

FIG. 5.-Definition of the five orbital contact times. In this illustration, the occulting screen has $\theta_{E}\left(t_{3}\right)<0, \dot{\theta}_{E}>0$, and $\dot{Y}_{E}>0$. 
constant light from star A. The total light exhibits "partial" or "diluted" eclipses, as were observed between the late 1960s and the early $1980 \mathrm{~s}$. After $t_{2}$, both stars undergo periodic eclipses, and the light curve can appear quite complex. Third contact $\left(t_{3}\right)$ occurs when the edge crosses the projected center of mass of the binary. There are no abrupt changes in the photometric behavior. After $t_{3}$, if the eccentricity is large, star B is seen for a rapidly shrinking fraction of the orbital period; it appears only briefly during the middle of each eclipse of star A. This is the era of "central rebrightenings," as observed in 1996 and 1997. After fourth contact $\left(t_{4}\right)$, the entire orbit of star B is hidden. As the screen advances further, the duration of the eclipses of star A increases until fifth contact $\left(t_{5}\right)$ when the entire system is covered.

\subsection{Interpretation of the Occulting Screen}

Of course, the real occulting edge is not semi-infinite. The dimensions of the screen's true sky projection depend on the threedimensional structure of the circumbinary disk. Star B should eventually reappear, possibly even before $t_{5}$, and the qualitative changes in total-light photometry that have been observed since 1960 may occur in reverse order as the trailing edge of the screen uncovers the binary. Those phenomena are not included in the model because the true three-dimensional disk structure is not known and cannot be determined from existing observations. However, some remarks are in order regarding the relation between the idealized screen and a real circumbinary disk.

The premise of the theory is that the circumbinary disk is inclined with respect to the binary plane, which causes the orbits of the disk's constituent particles to precess. Chiang \& MurrayClay (2004) showed that the disk should be warped and that its inner and outer radii should be of the same order of magnitude in order for the "ring" to precess as a unit. The edge of the screen may represent the ring's inner or outer edge in projection, or it may be a projection of the warp, in which case the edge does not necessarily represent a single orbital distance. These possibilities are depicted in Figure 6.

The correspondence between the precession of the disk and the motion of the screen is harder to visualize. For simplicity, imagine that the binary is surrounded by a circular ring of radius $a_{r}$ and inclination $I_{r}$ with respect to the binary's orbital plane. The ring represents, say, the inner radius of the disk, and its sky projection is the occulting feature. The ring's line of nodes (LON) precesses around the orbital plane with an angular frequency $\beta<0 .{ }^{13}$ Now imagine viewing the stellar orbits edge-on. When the LON of the ring is pointed at the observer, the ring appears as a straight line, with $Y_{E}=0$ and $\theta_{E}=I_{r}$. This is $t_{3}$, the moment of third contact. At other times, the sky projection of the ring is an ellipse. Considering the $Z>0$ half of the ellipse (i.e., the portion that is in front of the binary), the intersection point with the $Y$-axis and the angle it makes with the $X$-axis are

$$
\begin{gathered}
Y_{E}(t)=a_{r}\left[\frac{\tan I_{r} \sin \beta\left(t-t_{3}\right)}{\sqrt{1+\sin ^{2} \beta\left(t-t_{3}\right) \tan ^{2} I_{r}}}\right] \\
\theta_{E}(t)= \\
\tan ^{-1}\left\{\frac{\tan I_{r} \cos \beta\left(t-t_{3}\right)\left[\sin \beta\left(t-t_{3}\right) \cos I \tan I_{r}+\sin I\right]}{\sqrt{1+\cos ^{2} \beta\left(t-t_{3}\right) \tan ^{2} \beta\left(t-t_{3}\right)}}\right\} .
\end{gathered}
$$

\footnotetext{
13 The negative value of $\beta$ means that the LON regresses relative to the orbital angular momentum vector.
}

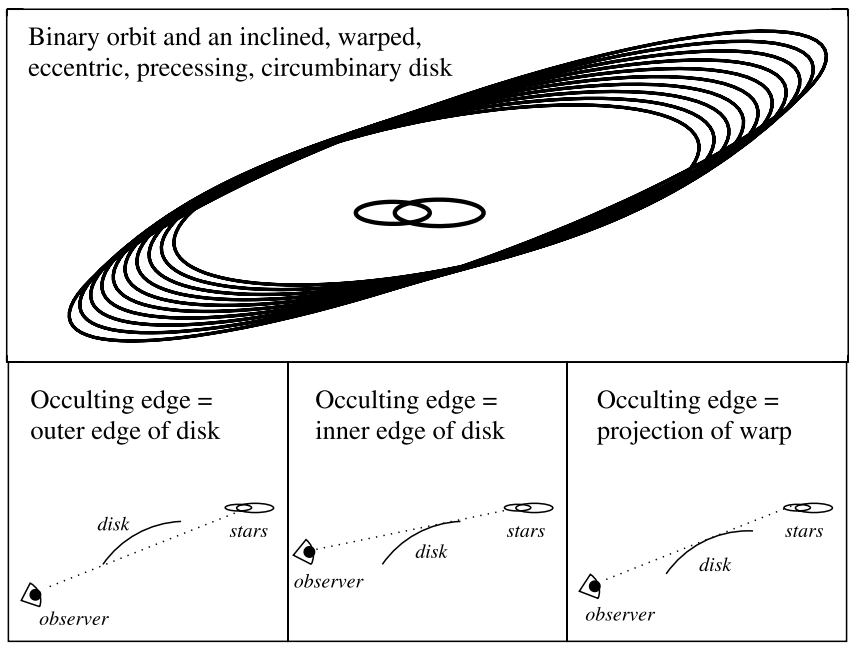

FIG. 6.- Illustration of the binary and the surrounding disk (top), and some possible viewing geometries (bottom). The true three-dimensional disk structure is not known. In particular the warp may have the opposite sign of the inclination gradient $\left(d I_{r} / d a_{r}\right)$ as the warp depicted. In the bottom panels, for clarity, only the cross section of the disk in the sky plane is shown.

For small $I_{r}$, these expressions reduce to $Y_{E}(t)=a_{r} \tan I_{r} \sin \beta(t-$ $\left.t_{3}\right)$ and $\theta_{E}(t)=I_{r} \cos \beta\left(t-t_{3}\right)$. For times close to $t_{3}, \dot{Y}_{E}(t)=$ $\beta a_{r} \tan I_{r}=$ constant and $\theta_{E}(t)=I_{r}=$ constant, in accordance with the assumptions made in Paper I.

However, the applicability of this approximation is not guaranteed. Over a 50yr time span, the higher order terms in $\beta\left(t-t_{3}\right)$ may be appreciable. More importantly, the disk is probably elliptical, given that the stellar binary has $e \sim 0.6$ (see Paper I; Hamilton et al. 2005; Herbst \& Moran 2005; and also $\S 5$ ). This alters both the amplitudes and phases of functions $Y_{E}(t)$ and $\theta_{E}(t)$, depending on the eccentricity and argument of pericenter of the ring. These functions may also depend on the details of the warp. In addition, if $a_{r}$ is small enough, the curvature of the occulting edge in the sky plane may be significant. We found that there are too many possibilities for the data to distinguish among them. Instead, our approach was to begin by pursuing the simplest possibility, a straight-edged screen with a constant angle and speed, while also preparing to allow for angular rotation speeds (of order $\sim \beta I_{r}$, according to the formula for a circular ring) and accelerations $\left(\sim \beta^{2} a_{r} \tan I_{r}\right)$ if they appear to be needed.

\subsection{Expectations for Parameter Values}

Before proceeding to the model-fitting results, we review what was already known of the parameter values from other observations or theoretical arguments. Star A has been spectrally classified as K6-7, with an effective temperature of approximately $4000 \mathrm{~K}$ and luminosity $0.4 L_{\odot}$ (Hamilton et al. 2001; Agol et al. 2004), assuming a cluster distance of 760 pc (Sung et al. 1997). Its mass was estimated to be $0.6 M_{\odot}$ by Flaccomio et al. (1999) and 0.5-1.0 $M_{\odot}$ by Hamilton et al. (2001), based on theoretical pre-main-sequence (PMS) evolutionary tracks. We find the range $M_{\mathrm{A}}=0.6 \pm 0.1 M_{\odot}$ to be in reasonable agreement with the six different sets of PMS tracks compiled by Hillenbrand \& White (2004). The corresponding radius estimate is $R_{\mathrm{A}}=1.3 \pm 0.1 R_{\odot}$. Unfortunately, we cannot estimate $M_{\mathrm{A}}$ and $R_{\mathrm{A}}$ independently using our model and the current data, because the limited radial velocity data place only weak bounds on the mass function of the binary and because there is a degeneracy between the stellar radius and the projected orbital speed during occultations. 
No spectrum of star B has been obtained, but its luminosity can be estimated using simple considerations of the system's photometric history. The rebrightenings observed between 1995 and 1998 (when star B was seen alone) had a maximum flux of 1.4 times the flux of the out-of-eclipse signal (when star A was seen alone), indicating that $L_{\mathrm{B}} / L_{\mathrm{A}} \approx 1.4$. The photographic photometry is not as accurate, but it also suggests $L_{\mathrm{B}} / L_{\mathrm{A}} \gtrsim 1$. The flux of the maximum-light phase of the pre-1980 photometry (when both stars were seen) was approximately double the flux of the minimum-light phase (when star A was seen alone) and was approximately 2.3 times that of the modern maximum-light phase. The PMS tracks of D'Antona \& Mazzitelli (1997) predict a mass-luminosity relation $L \propto M^{1.9}$ and a mass-radius relation $R \propto M^{0.27}$, leading to the expectations $M_{\mathrm{B}} / M_{\mathrm{A}} \approx 1.2$ and $R_{\mathrm{B}} / R_{\mathrm{A}} \approx 1.05$.

Johnson et al. (2004) analyzed the radial velocity data alone. Given the necessarily limited phase coverage of the observations, there were strong degeneracies between many of the orbital parameters. Among the most robust conclusions were that the orbital period is $P=48.4$ days, the eccentricity is appreciable $(e>0.3)$, and the argument of pericenter is within about $20^{\circ}$ of zero. The period determination is nearly identical with those based on periodograms of the photometry (by, e.g., Hamilton et al. 2005; Johnson \& Winn 2004; Maffei et al. 2005), although we note that the periodogram-based method may be biased because the light curve is not strictly periodic.

Herbst \& Moran (2005) and Hamilton et al. (2005) made a theoretical prediction for the eccentricity, based on the theory of tidal interactions in close binaries. Tides will "pseudosynchronize" the stellar spins and orbit, meaning that the stellar angular rotation frequency, $\Omega_{r}$, and the orbital angular frequency at pericenter, $n_{p}$, will reach a theoretically determined equilibrium (Hut 1981):

$$
\begin{aligned}
\frac{\Omega_{r}}{n_{p}} & =\frac{1+(15 / 2) e^{2}+(45 / 8) e^{4}+(5 / 16) e^{6}}{\left[1+3 e^{2}+(3 / 8) e^{4}\right](1+e)^{2}} \\
& =\frac{P_{\text {orb }}}{P_{\text {rot }}} \frac{\left(1-e^{2}\right)^{3 / 2}}{(1+e)^{2}} .
\end{aligned}
$$

The timescale for this process is poorly known but is estimated to be a few million years, which is approximately the age of the system. It therefore seems likely that pseudosynchronization is well underway, even if it has not yet been achieved. Hamilton et al. (2005) measured a rotation period $P_{\text {rot }}=9.6$ days for star A, from periodic photometric variations observed out of eclipse and attributed to star spots. Given that $P_{\text {orb }}=48.4$ days, the pseudosynchronization condition is $e=0.65 \pm 0.01$. It seems likely that the rotation of star A has been slowed down, rather than sped up, given that its period is longer than usual for a lowmass star in NGC 2264. If pseudosynchronization has not yet been achieved, then the equilibrium period must be even longer, and the eccentricity must be correspondingly smaller to provide a slower angular velocity at pericenter. Hence, whether or not pseudosynchronization has been achieved, the upper limit on the eccentricity is 0.66 . This assumes Hut's theory to be applicable, i.e., the effects of dynamical tides, and of forces from the circumbinary disk, accreting material, or other bodies in the system, can be neglected.

Some circumstantial evidence suggests that the orbital inclination is nearly $90^{\circ}$. First, the mere observation of eclipses suggests that the system is viewed nearly edge-on, unless the circumbinary disk is grossly misaligned with the orbital plane. Second, the inclination of star A's rotation axis $\left(I_{\star}\right)$ seems to be near $90^{\circ}$. The combination of the measured rotation period ( $P_{\text {rot }}=9.6$ days $)$,
TABLE 2

\begin{tabular}{|c|c|c|c|}
\hline Star & Year & Lower Bound & Upper Bound \\
\hline В & 1958 & 0.000 & 0.050 \\
\hline В & 1968 & 0.282 & 0.548 \\
\hline В & 1970 & 0.211 & 0.634 \\
\hline В & 1981 & 0.275 & 0.845 \\
\hline В & 1996 & 0.734 & 0.957 \\
\hline В & 1997 & 0.920 & 0.989 \\
\hline А & 1997 & 0.252 & 0.360 \\
\hline А & 1998 & 0.270 & 0.379 \\
\hline А & 1999 & 0.303 & 0.569 \\
\hline А & 2000 & 0.330 & 0.482 \\
\hline В & 2000 & 1.000 & 1.000 \\
\hline А & 2001 & 0.326 & 0.550 \\
\hline А & 2002 & 0.410 & 0.432 \\
\hline А & 2003 & 0.444 & 0.474 \\
\hline А & 2004 & 0.497 & 0.539 \\
\hline 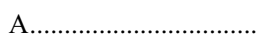 & 2005 & 0.530 & 0.570 \\
\hline
\end{tabular}

Observed Bounds on Eclipse Fractions

the projected rotation speed of the photosphere $\left(V_{\text {rot }} \sin I_{\star}=\right.$ $\left.6.9 \pm 0.3 \mathrm{~km} \mathrm{~s}^{-1}\right)$, and the estimated stellar radius $\left(R_{\mathrm{A}}=1.3 \pm\right.$ $\left.0.1 R_{\odot}\right)$ gives $\sin I_{\star}=1.01 \pm 0.09$, or $I_{\star}>67^{\circ}$. Third, Hamilton et al. (2003) observed a double-peaked emission line of [O I], with the peaks at radial velocities $\pm 20 \mathrm{~km} \mathrm{~s}^{-1}$ of the systemic velocity. These are thought to arise from bipolar jets with space velocities that are typically $\pm 200 \mathrm{~km} \mathrm{~s}^{-1}$, suggesting that the jets (and by implication the stellar poles and the orbit) are inclined by $\gtrsim 80^{\circ}$.

Finally, an expectation for $\gamma$ (the heliocentric radial velocity of the center of mass) is available thanks to the radial velocity survey of NGC 2264 by Soderblom et al. (1999). These authors found a median radial velocity of $20 \pm 3 \mathrm{~km} \mathrm{~s}^{-1}$ among probable cluster members. Thus, although Johnson et al. (2004) found the radial velocity measurements to be compatible with any value of $\gamma$ between 7 and $22 \mathrm{~km} \mathrm{~s}^{-1}$, we expect the true value of $\gamma$ to be near the high end of that range, assuming $\mathrm{KH} 15 \mathrm{D}$ to be a cluster member.

\section{MODELS OF THE ORBIT}

We take a two-step approach to the quantitative modeling of KH 15D. The first step is a direct continuation of Paper I. Rather than fitting the photometry directly, we attempt to match some of its most important properties: the eclipse durations and the ingress and egress durations. For the moment, we ignore all of the other information in the photometry, such as the light observed during eclipses, and the detailed shapes of the ingress and egress light curves. The second step, described in $\S 6$, is an attempt to fit the full photometric time series.

To measure the eclipse durations, we plotted the phased light curve for each year in which fairly complete phase coverage was achieved and determined the minimum and maximum phases of the half-flux points of the eclipses. The results are given in Table 2. They agree with those given in Paper I and include new measurements based on the newly available photometry, as well as a few measurements of the eclipse fraction of star B based on the observations of central rebrightenings (or lack thereof).

The ingress and egress durations are harder to measure because fine time sampling is needed. In Paper I we estimated the durations based on phased light curves from 2001-2003, but we see now that there is too much variation between eclipses for this method to be reliable. Instead, we used data from a single eclipse in 2002.1 that was observed with especially high cadence. We considered only the steepest (most rapidly varying) portion of 

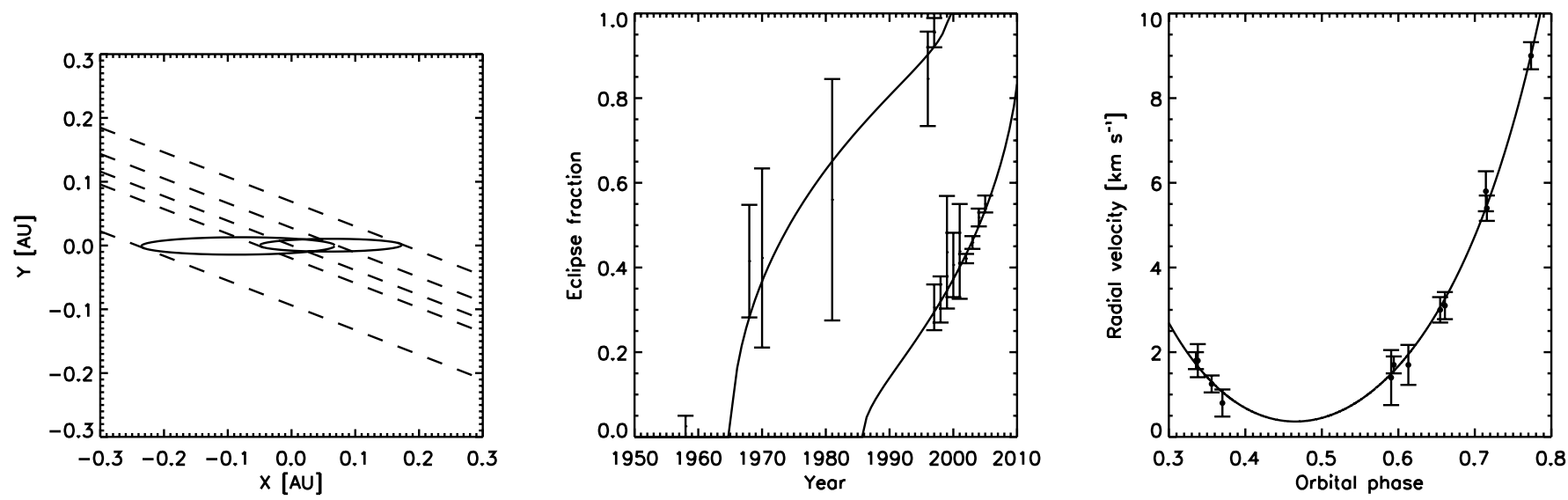

FIg. 7.- Model 1: $M_{\mathrm{B}}$ is a free parameter and $\dot{\theta}_{E}=0$. Left: Binary orbit (solid lines) and position of the occulting edge at the five orbital contact times (dashed lines). Center: The eclipse fractions of stars A and B as a function of time, as observed (error bars) and calculated (solid lines). Right: Radial velocity variations of star A as a function of orbital phase, as observed (points with error bars) and calculated (solid line).

the light curve between flux levels $33 \%$ and $67 \%$ of the maximum and fitted a model light curve to these data consisting of a limb-darkened star being crossed by a knife-edge occulter. The best-fitting ingress and egress durations (the times taken to cross the diameter of the star) were $2.55 \pm 0.08$ and $2.96 \pm 0.05$ days, respectively.

We assume $M_{\mathrm{A}}=0.6 M_{\odot}$ and $R_{\mathrm{A}}=1.3 R_{\odot}$ as explained in $\S 4.3$. The mass of star $B$ is taken to be a free parameter. There are six free parameters describing the orbit: $\left\{P, e, I, \omega, \gamma, T_{p}\right\}$. Additional free parameters are needed to describe the occulting screen and its trajectory. We begin with the case of a screen that moves uniformly with a fixed angle, in which case three parameters are needed. One obvious parameterization, for example, is $\left\{Y_{E}\left(T_{p}\right), \dot{Y}_{E}, \theta_{E}\right\}$. We use the alternative parameter set $\left\{t_{4}, t_{5}, \theta_{E}\right\}$, which provides a more direct connection to the observations.

The figure-of-merit function is

$$
\begin{aligned}
\chi^{2} & =\chi_{v}^{2}+\chi_{d}^{2} \\
& =\sum_{i=1}^{N_{v}}\left(\frac{v_{C, i}-v_{O, i}}{\sigma_{v, i}}\right)^{2}+\sum_{i=1}^{N_{d}}\left(\frac{d_{C, i}-d_{O, i}}{\sigma_{d, i}}\right)^{2},
\end{aligned}
$$

where $v_{O, i}$ are the observed radial velocities (of which there are $\left.N_{v}=12\right), \sigma_{v, i}$ are the corresponding $1 \sigma$ uncertainties, and $v_{C, i}$ are the radial velocities as calculated according to the model. The single measurement of the ingress duration $\left(d_{C, 1}\right)$ and the egress duration $\left(d_{C, 2}\right)$ are described with a similar notation. We used an AMOEBA algorithm (Press et al. 1992, p. 408) to minimize $\chi^{2}$, while simultaneously requiring the calculated eclipse durations to obey the 16 constraints ${ }^{14}$ given in Table 2. We also enforced the proper phase alignment between the radial velocity data and the photometry by requiring the model system to experience mideclipse within 5 days of JD 2,452,352.5, a particular mideclipse observed in 2002.

An excellent fit is achieved, with $\chi^{2}=3$ and 4 degrees of freedom (dof). Thus, the data are simultaneously consistent with the measurements of radial velocity, ingress and egress duration, and eclipse duration. Figure 7 shows the fit to the radial velocity and eclipse-fraction data. It also shows a diagram of the orbit and

\footnotetext{
${ }^{14}$ The eclipse duration measurements have the character of strict bounds, rather than central values and $1 \sigma$ uncertainties, because the measurements are limited by time-sampling of the events rather than statistical errors in the flux measurements.
}

the screen at each of the five orbital contact times. The bestfitting parameter values are given in Table 3 under the heading "Model 1."

Many of the best-fitting parameter values conform with the expectations given in $\S 4.3$ : the period is $P=48.4$ days, the eccentricity is $e=0.55$, the inclination is $83^{\circ}$, and the argument of pericenter is $6^{\circ}$. However, there are two exceptions. First, the heliocentric radial velocity of the center of mass $\left(14 \mathrm{~km} \mathrm{~s}^{-1}\right)$ is somewhat low; KH 15D would be a $\sim 2 \sigma$ outlier in the radial velocity distribution of the cluster. A second and much more

TABLE 3

\begin{tabular}{|c|c|c|c|}
\hline \multirow[b]{2}{*}{ Parameter } & \multicolumn{3}{|c|}{ Best-Fitting VALUe } \\
\hline & Model 1 & Model 2 & Model 3 \\
\hline$M_{\mathrm{A}}\left(M_{\odot}\right) \ldots$ & 0.6 & 0.6 & $0.6 \pm 0.1$ \\
\hline$M_{\mathrm{B}} / M_{\mathrm{A}}$ & 0.76 & 1.2 & $1.2 \pm 0.1$ \\
\hline$L_{\mathrm{A}}\left(L_{\odot}\right)$ & $\ldots$ & $\ldots$ & $0.413 \pm 0.006$ \\
\hline$L_{\mathrm{B}} / L_{\mathrm{A}} \ldots \ldots \ldots \ldots \ldots \ldots \ldots \ldots \ldots \ldots \ldots \ldots \ldots$ & $\ldots$ & $\ldots$ & $1.36 \pm 0.10$ \\
\hline$L_{0} / L_{\mathrm{A}}$ & $\ldots$ & $\ldots$ & $0.029 \pm 0.005$ \\
\hline$R_{\mathrm{A}}\left(R_{\odot}\right)$ & 1.3 & 1.3 & $1.30 \pm 0.07$ \\
\hline$R_{\mathrm{B}} / R_{\mathrm{A}}$ & 1.05 & 1.05 & $1.05 \pm 0.03$ \\
\hline$\xi_{1}$ & $\ldots$ & $\ldots$ & $2.0 \pm 0.3$ \\
\hline$\xi_{2}$ & $\ldots$ & $\ldots$ & $3.3 \pm 0.4$ \\
\hline$\epsilon_{1}$ & $\ldots$ & $\ldots$ & $0.25 \pm 0.05$ \\
\hline$\epsilon_{2}$ & $\ldots$ & $\ldots$ & $0.049 \pm 0.003$ \\
\hline$P$ (days) & 48.4 & 48.4 & $48.381 \pm 0.005$ \\
\hline 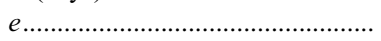 & 0.55 & 0.58 & $0.574 \pm 0.017$ \\
\hline$I(\operatorname{deg})$ & 83 & 83 & $92.5 \pm 2.5$ \\
\hline$\omega(\operatorname{deg})$ & 6 & 14 & $13 \pm 2$ \\
\hline$T_{p}(\mathrm{JD})-2,452,350 \ldots \ldots \ldots \ldots \ldots \ldots$ & 1.1 & 0.7 & $1.9 \pm 0.7$ \\
\hline$\gamma\left(\mathrm{km} \mathrm{s}^{-1}\right)$ & 14.1 & 18.4 & $18.6 \pm 1.3$ \\
\hline$t_{1} \ldots \ldots \ldots \ldots \ldots$ & 1965 & 1963 & $1962 \pm 3$ \\
\hline$t_{2} \ldots \ldots \ldots$ & 1985.3 & 1985.9 & $1985.9 \pm 0.7$ \\
\hline 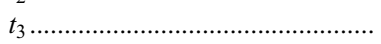 & 1991.1 & 1992.5 & $1992.7 \pm 0.5$ \\
\hline$t_{4} \ldots \ldots \ldots \ldots$ & 1998.8 & 1997.0 & $1997.3 \pm 0.5$ \\
\hline 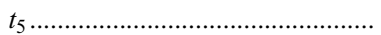 & 2010.9 & 2008.0 & $2008.0 \pm 0.2$ \\
\hline$\theta_{E}\left(t_{4}\right)(\operatorname{deg}) \ldots \ldots \ldots \ldots \ldots \ldots \ldots \ldots \ldots \ldots$ & -20 & -16 & $-32 \pm 7$ \\
\hline 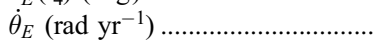 & 0 & 0.0061 & $0.0087 \pm 0.0015$ \\
\hline
\end{tabular}

KH 15D Model Parameters

Notes.-Models 1 and 2 were fitted to the eclipse duration, ingress/egress duration, and radial velocity data. In model $1 M_{\mathrm{B}}$ is a free parameter and $\dot{\theta}_{E}=0$. In model $2 M_{\mathrm{B}} / M_{\mathrm{A}}=1.2$ and $\theta_{E}$ is a free parameter. Model 3 was fitted to the photometric and radial velocity data. The uncertainties were determined with the Monte Carlo procedure described in the text. 

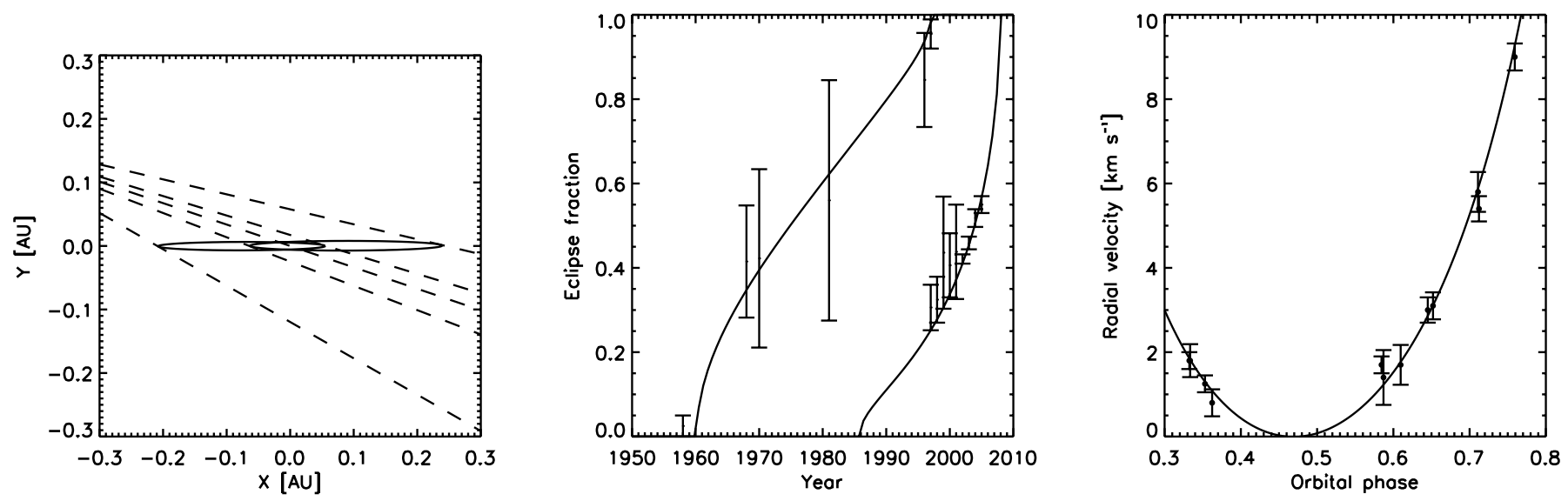

FIG. 8. - Model 2: $M_{\mathrm{B}} / M_{\mathrm{A}}=1.15$ and $\dot{\theta}_{E}$ is a free parameter. The plotting conventions are the same as in Fig. 7.

serious problem is that the mass ratio is $M_{\mathrm{B}} / M_{\mathrm{A}}=0.76$, which is less than unity, even though it seems certain that $L_{\mathrm{B}} / L_{\mathrm{A}}>1$.

The latter problem can be traced to the assumption that the occulting screen moves uniformly in a constant direction. Under this assumption, $t_{5}-t_{2}$ is proportional to the projected size of star A's orbit, which in turn is proportional to $M_{\mathrm{A}}^{-1}$. Likewise, $t_{4}-t_{1} \propto M_{\mathrm{B}}^{-1}$. The photometry provides the constraints $1959<$ $t_{1}<1967,1981<t_{2}<1996,1997<t_{4}<2000$, and $t_{5}>2005$, and an extrapolation of the rapidly rising eclipse fraction suggests $t_{5}<2010$. Together, these inequalities give

$$
\frac{M_{\mathrm{B}}}{M_{\mathrm{A}}}=\frac{t_{5}-t_{2}}{t_{4}-t_{1}}<0.97
$$

(assuming constant screen velocity). If $M_{\mathrm{B}} / M_{\mathrm{A}}$ is held fixed at 1.2 , the value expected from a consideration of the luminosity ratio, then it is impossible to fit the eclipse data. For example, in the model, star B is covered $6 \mathrm{yr}$ too early, or the rise in the eclipse fraction of star A over the last decade is much slower than has been observed.

As explained in $\S 4.2$, one might expect the real projection of a circumbinary disk to exhibit deviations from a constant velocity. Once the screen is allowed to rotate or accelerate during its passage in front of the binary orbit, a much wider range of mass ratios is allowed. While this saves the model from a seemingly unphysical prediction, it becomes impossible to determine the stellar mass ratio independently. Instead, in what follows, we hold the mass ratio fixed at $M_{\mathrm{B}} / M_{\mathrm{A}}=1.2$. Because the leading-order correction in the precession angular velocity $\beta$ is a rotation, we concentrate on the case of uniform motion plus a uniform rotation (with no acceleration). After dropping one of the original free parameters affecting the radial velocities $\left(M_{\mathrm{B}}\right)$, and adding a new one $\left(\dot{\theta}_{E}\right)$ affecting only the eclipses, the resulting fit is also good, with $\chi^{2}=6$ and 5 dof. The fit to the data is shown in Figure 8, and the best-fitting parameter values are in Table 3 under the heading "Model 2."

We conclude that it is possible to fit the data with a physically plausible mass ratio. All of the parameters agree with the expectations given in $\S 4.3$, including the heliocentric radial velocity $\left(\gamma=18.4 \mathrm{~km} \mathrm{~s}^{-1}\right)$. The best-fitting value for the rotation rate is $\dot{\theta}_{E}=6 \times 10^{-3} \mathrm{rad} \mathrm{yr}^{-1}$, which is compatible with reasonable guesses for the circumbinary disk properties. It implies a precession rate of order $\beta \sim 10^{-2} \mathrm{rad} \mathrm{yr}^{-1}$, as would be experienced by a circular ring of radius $\sim 3$ AU. However, a perfectly circular ring is not realistic on physical grounds and is not consistent with the data beyond this order-of-magnitude comparison; the rota- tion rate in that case would be exactly zero at $t_{3}$, in contradiction to the assumptions of the model. We also found that a uniform acceleration with no rotation provides a reasonable fit, and obviously a combination of rotation and acceleration is possible. Given the uncertainty in both the stellar mass ratio and the geometry of the disk, we cannot use the model alone to decide which of these cases is closer to the truth.

\section{MODELS OF THE OCCULTATIONS}

The models described in the previous section ignore some potentially interesting and information-bearing aspects of the photometry, such as the phase of each individual eclipse, the shape of the ingress and egress light curves, and the progressive deepening of the eclipses. In this section we elaborate on our model in several ways and attempt to fit all of the photometry.

In Paper I, we generated model light curves by treating the occulting feature as a completely opaque and semi-infinite knifeedge, and the stars as limb-darkened photospheres. Although these model light curves successfully reproduce the basic patterns in the photometry, there are at least three ways in which they fail to match the data within the measurement uncertainties:

1. The model does not reproduce the observed $\sim 0.1 \mathrm{mag}$ variations outside of eclipses. As noted previously, Hamilton et al. (2005) provided evidence for quasiperiodic variability outside of eclipses and interpreted the 9.6 day period as the stellar rotation period of star A. Presumably, these episodic variations are caused by cool star spots that last only a few months, as is common for young low-mass stars (see, e.g., Herbst et al. 1994).

2. The steepest (central) phase of each occultation light curve is consistent with a knife-edge crossing a photosphere. However, during the first and last third of each event, the flux variation is slower than such a model would predict, as noted by Agol et al. (2004) and in Paper I.

3. Light is observed even during mideclipse, when (according to the model) the photospheres of both stars are completely hidden. The mideclipse flux has fallen from about $9 \%$ to $2 \%$ of the uneclipsed flux over the last decade (see Fig. 2). Indeed, the mideclipse light is not constant even within a single eclipse. Although the rebrightening events have diminished dramatically in intensity since 1998, there are still faint echoes of this phenomenon in the more recent data.

We deal with the first point by treating the episodic variations of star A as an intrinsic source of noise. We do not expect the light curve to be predictable with better than 0.1 mag accuracy. As for the second and third points, we have found that a good fit 

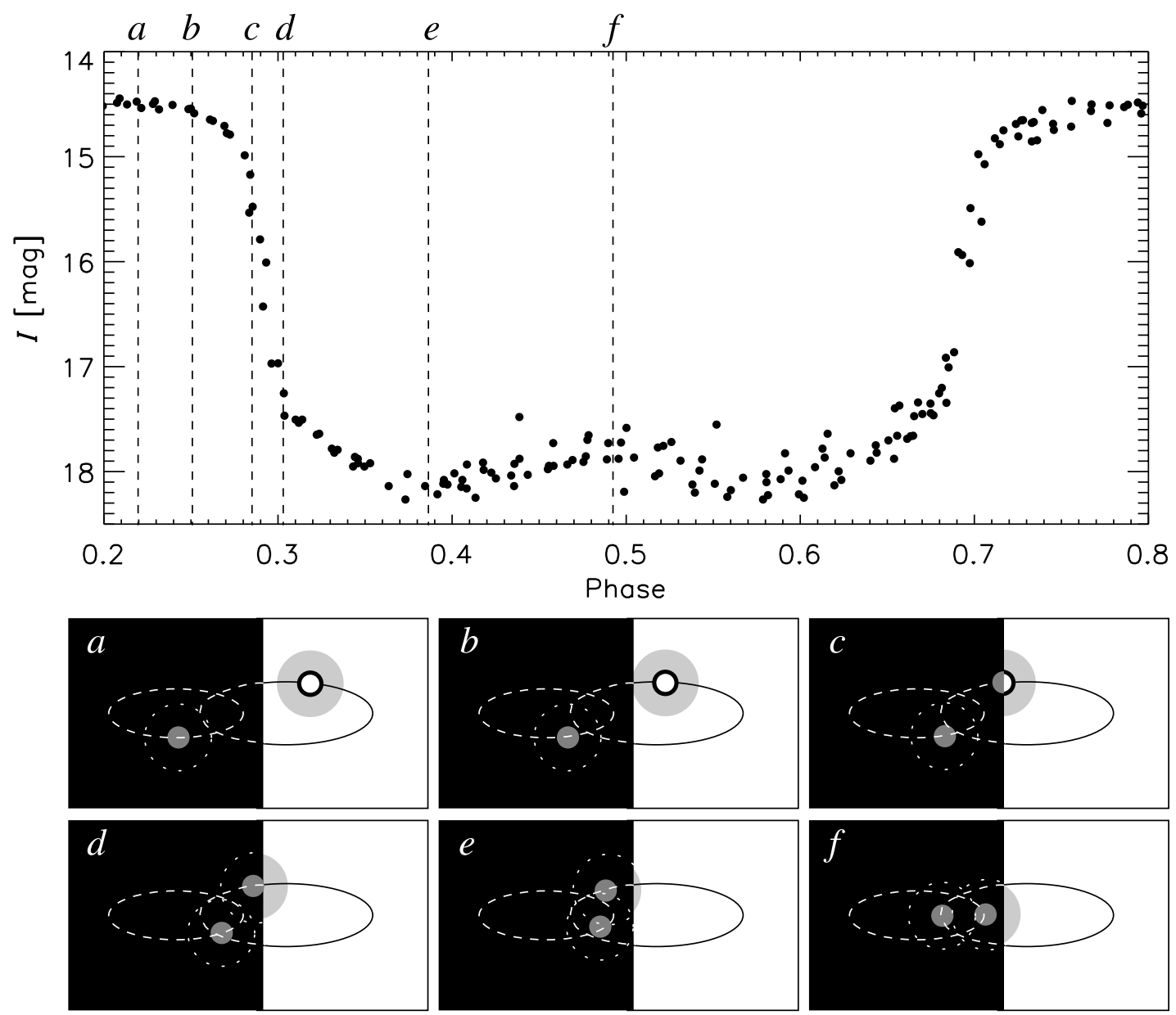

FIg. 9.- - Illustration of the model for the occultation light curve. Top: Phased light curve from the 2001-2002 season, with six particular phases marked with vertical dashed lines. Bottom: Corresponding configurations of the stars, halos, and the occulting edge. These are cartoons only, and do not represent optimized model parameters. In particular, the best-fitting model halos are asymmetric unlike the circular halos drawn here.

is achieved with a simple model in which each star is enveloped by a faint halo. Figure 9 illustrates how this model accounts for the light observed during eclipses, the intraeclipse variations, and the shape of the ingress and egress light curves. The dashed lines mark six special photometric phases (labeled " $a$ " " $f$ ") for which the configurations of the photospheres, the halos, and the occulting screen are illustrated in the smaller panels beneath the light curve. These phases are: $(a)$ Out of eclipse. Both the photosphere and the halo of star A are seen. (b) Start of ingress. The halo of star A begins to be occulted. (c) Midpoint of ingress. The photosphere is half-covered and the total flux declines steeply. (d) The photosphere is completely occulted, and the total flux resumes its shallower decline. (e) The halo of star A is almost completely covered, and the total flux reaches a local minimum. ( $f$ ) Star B reaches its closest approach to the occulting edge, and its halo is maximally exposed. The total flux reaches a local maximum. (At this phase, before $t_{4}$, the photosphere of star B was exposed and manifested as the rebrightening event.) Although it is not illustrated in Figure 9, the gradual darkening of the mideclipse phase can also be accounted for with this model: as the occulting edge advances, the fraction of the halo of star B that is exposed at mideclipse $(f)$ gradually shrinks.

To develop a quantitative model, we first demonstrate that the occultations of star A are repeatable events: all of the observed ingress and egress events are nearly equivalent, after correcting for the differences in orbital velocity of the star at the contact points with the occulting edge. Using our model, we calculate the position of star A and of the occulting edge as a function of time. Then, instead of plotting the flux versus time, we plot the flux versus the position of the occulting edge relative to star A. For this purpose it is convenient to use a rotated coordinate system $(x, y)$, in which the occulting edge is the line $x=x_{E}(t)$, the perpendicular direction is the $y$-axis, and distances are measured in units of $R_{\mathrm{A}}$. The sky position of star A is $\left(x_{\mathrm{A}}, y_{\mathrm{A}}\right)$. The top panel of Figure 10 shows the result, summarizing $10 \mathrm{yr}$ of photometry as star A goes back and forth beneath the occulting edge. The measured flux is indeed a coherent function of $\Delta x \equiv x_{E}-x_{\mathrm{A}}$, with a scatter of $\sim 10 \%$.

This allows us to model the occultations as a knife edge scanning over a time-invariant surface brightness distribution associated with the star, $S\left(x-x_{\mathrm{A}}, y-y_{\mathrm{A}}\right)$. Since the relative velocity of the edge and the star is always closely aligned with the $x$-axis, the only relevant quantity is the one-dimensional brightness distribution

$$
B(x)=\int_{-\infty}^{\infty} d y S(x, y) .
$$

When the occulting edge is at $x_{E}$ and star $\mathrm{A}$ is at $\left(x_{\mathrm{A}}, y_{\mathrm{A}}\right)$, the measured flux is the cumulative one-dimensional brightness distribution

$$
C=\int_{x_{E}}^{+\infty} d x B\left(x-x_{\mathrm{A}}\right)=\int_{\Delta x}^{+\infty} d x B(x),
$$




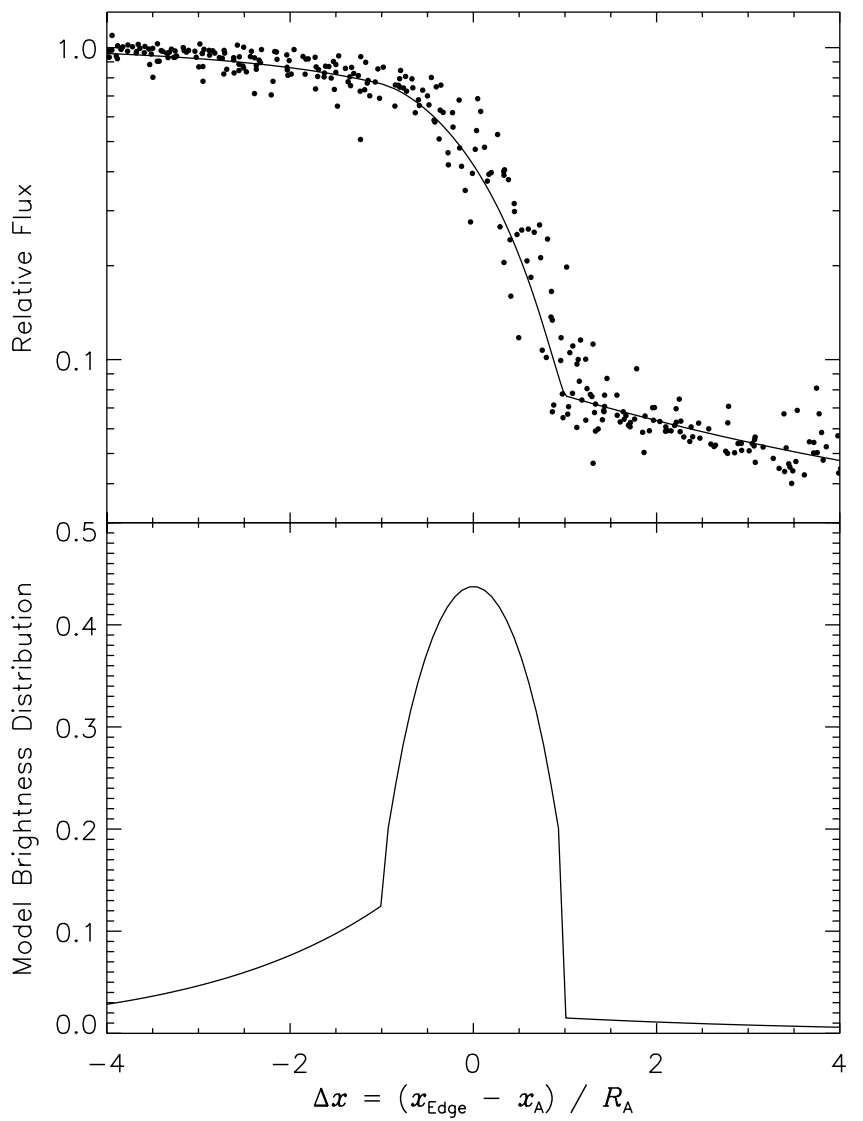

FIG. 10.-Top: Relative flux of KH $15 \mathrm{D}$ as a function of the position of the occulting edge. The quantity $\Delta x$ is the sky-projected distance between the edge and the center of star A, in units of the stellar radius. For a perfectly sharp and straight edge, this plot can be interpreted as the cumulative one-dimensional brightness distribution of the star and its apparent halo. The dots show the data, and the solid line is the best-fitting model. Bottom: One-dimensional brightness distribution of the best-fitting model.

which is a function of $\Delta x$, as observed. We note that $B$ represents all of the flux that moves with star A on the sky. It includes light from the star, any luminous material that orbits with the star, and also any scattering halo (which we argue later does exist). The halo need not be physically associated with the star, just as the lunar halo that is sometimes observed on cold nights is not physically associated with the Moon.

Next, we devise a fitting function for $B$ that is consistent with the top panel of Figure 10. At $\Delta x=0$, the occulting edge intersects the center of star A, and approximately one-half of the total flux is exposed. As the screen advances over the star from $\Delta x=0$ to 1 , the received flux falls rapidly, in a manner consistent with a knife edge covering a stellar photosphere. The abrupt slope change at $\Delta x=1$ is suggestive of the contact between a sharp occulting edge and the rim of the stellar photosphere. For $\Delta x>1$, the flux falls off much more gradually, as if the star is trailed by a spatially extended halo comprising a few percent of the total flux. Interestingly, the light curve is not symmetric about $\Delta x=0$. Once the star is more than half exposed $(\Delta x<0)$, the flux rises more gradually than one would expect for a symmetric halo. The side of the star nearest to the occulting edge appears more smeared out than the other side.

After some experimentation, we found good results for the fitting function

$$
B(x)= \begin{cases}B_{1} \exp \left[(x+1) / \xi_{1}\right], & x \leq-1 \\ B_{1}+B_{\star}(x), & -1<x<1 \\ B_{2} \exp \left[-(x-1) / \xi_{2}\right], & x \geq 1 .\end{cases}
$$

The ingredients of this function are the brightness distribution of the stellar photosphere $\left(B_{\star}\right)$, and an exponential falloff on each side of the star. The amplitude $\left(B_{1}\right.$ or $\left.B_{2}\right)$ and scale length $\left(\xi_{1}\right.$ or $\left.\xi_{2}\right)$ of the exponential function are allowed to be different on each side, to match the observed asymmetry in $C$, and the function is discontinuous at $x=1$, to match the observed slope discontinuity in $C$. In practice, rather than quoting $B_{1}$ and $B_{2}$, it is more useful to describe the brightness distribution by the total flux $(L)$ and the fraction of the total flux carried by each side of the exponential halo $\left(\epsilon_{1}\right.$ and $\left.\epsilon_{2}\right)$. Although the photosphere may be modeled as uniform to within the accuracy of the data, for completeness we use a linear limb-darkening law, with a coefficient of $0.65 .{ }^{15}$ We emphasize that equation (12) is merely a fitting formula. In fact, we argue later that the halo is not a physical object, but rather represents forward- or back-scattering by particles in the circumbinary disk. In this section, we limit ourselves to demonstrating the success of the phenomenological "halo model," deferring further discussion of the physical interpretation until $\S 7$.

Next, we proceed to the optimization of parameters. As before, we assume $M_{\mathrm{A}}=0.6 M_{\odot}, R_{\mathrm{A}}=1.3 R_{\odot}$, and $M_{\mathrm{B}} / M_{\mathrm{A}}=$ 1.2. The occulter is an opaque, semi-infinite straight edge whose intersection point $Y_{E}(t)$ and rotation angle $\theta_{E}(t)$ are both linear functions of time. Both star A and star B are modeled with the function given in equation (12), and we add a time-invariant term $L_{0}$, representing any large-scale (unocculted) components of the surface-brightness distribution. All together, the model has $N_{p}=$ 17 free parameters: 7 specifying the surface-brightness distribution, $\left\{L_{\mathrm{A}}, L_{\mathrm{B}}, L_{0}, \epsilon_{1}, \epsilon_{2}, \xi_{1}, \xi_{2}\right\} ; 6$ specifying the orbit, $\left\{P, e, I, \omega, T_{p}, \gamma\right\}$; and 4 describing the occulting screen, $\left\{t_{4}, t_{5}, \theta_{E}\left(t_{4}\right), \dot{\theta}_{E}\right\}$.

It was desirable to speed up the computations by time-averaging the photometry. Whenever more than one measurement was made within a given $6 \mathrm{hr}$ time period, we computed the fluxweighted mean of the results. The resulting binned data set has $N_{f}=1044$ entries. The fitting statistic is

$$
\chi^{2}=\sum_{i=1}^{N_{f}}\left(\frac{f_{C, i}-f_{O, i}}{\sigma_{f, i}}\right)^{2}+\lambda \sum_{i=1}^{N_{V}}\left(\frac{V_{C, i}-V_{O, i}}{\sigma_{V, i}}\right)^{2}
$$

which is analogous to equation (8), with the fluxes $f$ replacing the ingress or egress durations $d$. The uncertainty $\sigma_{f, i}$ was taken to be the quadrature sum of the measurement uncertainty and $10 \%$, where the latter is intended to account for intrinsic variations. The multiplier $\lambda$ controls the relative weighting of the flux data and radial velocity data. If the measurement uncertainties were known perfectly and the model were correct, then $\lambda=1$ would be the appropriate choice and the best-fitting solution would have $\chi^{2} \approx 1040=N_{f}+N_{V}-N_{p}$.

In this case $\lambda=1$ is probably not the best choice. We expect the model to be statistically consistent with the radial velocity data, because the only relevant aspect of the model — the assumption of a fixed Keplerian orbit - seems reasonable. In contrast, the photometric model involves idealized assumptions about the occulter and the surface-brightness distribution, and the estimates of $\sigma_{f}$ are crude. Our approach was to force the model to be statistically consistent with the radial velocity data, by increasing $\lambda$ until

\footnotetext{
${ }^{15}$ For a star with $T_{\text {eff }} \approx 4000 \mathrm{~K}$ and $g=10^{4} \mathrm{~cm} \mathrm{~s}^{-2}$, a linear limb-darkening coefficient of $u \approx 0.65$ is predicted by Claret $(1998,2000)$ for the Cousins $I$ band. Van Hamme (1993) predicts a similar value, 0.6 . The value of 0.3 used by Herbst et al. (2002) and in Paper I was chosen mistakenly. In any case, the choice of the limb-darkening coefficient has a very minor effect on the model light curve.
} 

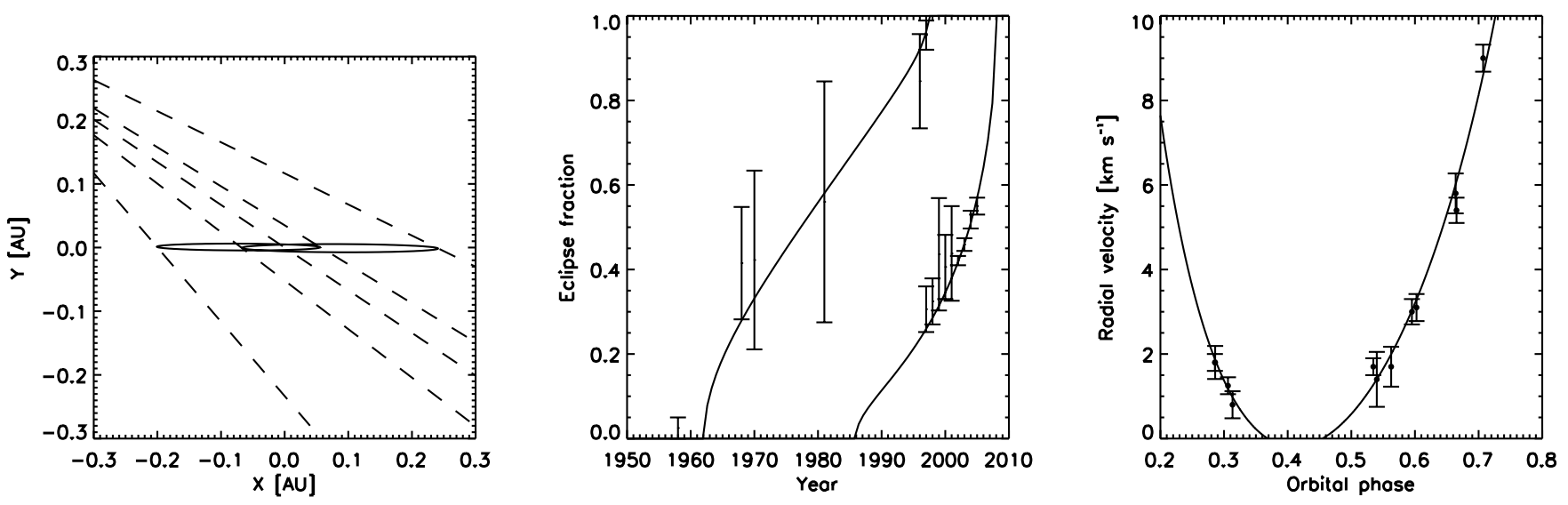

FIG. 11.-Model 3: $M_{\mathrm{B}} / M_{\mathrm{A}}=1.2, \dot{\theta}_{E}$ is a free parameter, and the full photometric time series is fitted (rather than just the eclipse fractions and partial-eclipse durations). The plotting conventions are the same as in Fig. 7.

the best-fitting solution had $\chi_{V}^{2} \approx 12=N_{V}$. Of course, we cannot be certain that the radial velocity data are free from additional sources of measurement error and unmodeled systematic effects, such as precession of the orbit due to perturbations from the circumbinary disk and additional bodies. Nevertheless, in the rest of this section, we describe the optimized model that results from this procedure.

With $\lambda=50$, the best-fitting model has $\chi_{V}^{2}=13$ and $\chi_{f}^{2}=$ 1574. The orbit and the fit to the radial velocity data are shown in Figure 11. The fit to the photometry is shown in Figure 12. Each panel shows the phased light curve for a particular time interval. The time intervals chosen for this plot are the intervals that are marked by dashed lines in Figure 2. The final column of Table 3, under the heading "Model 3," gives the best-fitting parameters.

The table includes estimated uncertainties in the fitted quantities, which should be treated with caution. They were calculated using a Monte Carlo algorithm, as follows. We optimized the parameters on each of $10^{4}$ synthetic data sets ("realizations"). Each realization consisted of $N_{f}=1044$ flux measurements and $N_{V}=$ 12 radial velocity measurements (the same numbers as the real data set). The flux measurements were randomly selected from the real data set, with repetitions allowed. The intent is to estimate the probability distribution of the measurements using the measured data values themselves (see, e.g., Press et al. 1992, p. 689). The number of radial velocity measurements is too small for drawing with replacement to be effective. Instead, we added Gaussian random numbers to the measured radial velocities, with a mean of zero and a standard deviation given by the quoted $\sigma_{v}$ values. To account for the uncertainty in the stellar masses, we assigned $M_{\mathrm{A}} / M_{\odot}$ by picking a random number from a Gaussian distribution with mean 0.6 and standard deviation 0.1 . Likewise, for the mass ratio $M_{\mathrm{B}} / M_{\mathrm{A}}$, we used a Gaussian distribution with a mean of 1.2 and a standard deviation of 0.1 . The stellar radii were fixed according to the relation $R \propto M^{0.27}$. For each parameter, we found the median and the approximate $68 \%$ confidence limits in the resulting distribution of best-fitting values.

There are many caveats. To begin with, the model is not in formal statistical agreement with the data (some discrepancies are highlighted below). The random flux variations of the stars are not independent; they are correlated on timescales of days. The a priori probability distributions for the stellar masses are probably not Gaussian. The systematic error related to our assumption about the trajectory of the occulting edge has not been taken into account. One can imagine more complex analyses that attempt to overcome these problems, but we do not believe that such methods are justified.

The model shares the successes of the models presented in $\S 5$. It reproduces the transitions from no eclipses to partial eclipses to total eclipses, the existence of the central reversals from at least 1995 until 1999, and the growing duty cycle of the modern eclipses. The more detailed photometric model also accounts for the relative phases of the partial and total eclipses, the gradual deepening of the eclipses and the slope discontinuities in the ingress and egress light curves. In addition to fitting the data reasonably well, the model satisfies all of the criteria that were discussed in $\S 4.3$. The stellar masses, radii, and luminosities are reasonable, by fiat. The orbital eccentricity is smaller than the theoretical upper bound of 0.66 , and the orbital inclination is close to edge-on. The heliocentric radial velocity of the center of mass is near the median that has been observed among cluster members. The screen trajectory is consistent with order-ofmagnitude estimates for the expected motion of the edge of a precessing circumbinary ring with a radius of $\sim 3 \mathrm{AU}$.

The best-fitting brightness distribution for star A is shown in Figure 10. The top panel shows the model cumulative onedimensional brightness distribution overlaid on the data points, while the bottom panel shows the model brightness distribution itself. The asymmetry of the halo is evident. The exponential scale length on both sides is a few stellar radii, but the fractional flux of the halo is much greater on the side closest to the occulting edge ( $\sim 25 \%)$ than on the other side $(\sim 5 \%)$.

As noted before, the model cannot reproduce the apparently random $\sim 10 \%$ flux variations of star A that occur on timescales of a few days. Nor can it reproduce the seasonal fluctuations of a few percent in the mean flux of star A. There are some other significant discrepancies between the data and the model. In the 1967-1972 time period, there is a $\sim 0.1$ mag offset between the calculated and observed light curves, and, in addition, the calculated eclipse depth is $\sim 0.15 \mathrm{mag}$ too deep. These problems are at least partly the fault of the uncertainty in the photographic magnitude scale. The offset is within the 0.14 mag uncertainty in the zero point, and the calculated depth becomes too shallow if we use the $I_{\mathrm{M}}$ system instead of the $I_{\mathrm{J}}$ system (see $\S 2.1$ ). Another discrepancy is the phase shift of $\sim 0.02$ between some of the calculated and observed central rebrightenings. This is most easily seen in the 2000-2001 data. This offset disappears for an orbital period of 48.36 days, but that value of $P$ is disfavored by the radial velocity data and by the relative phases of the pre- 1980 


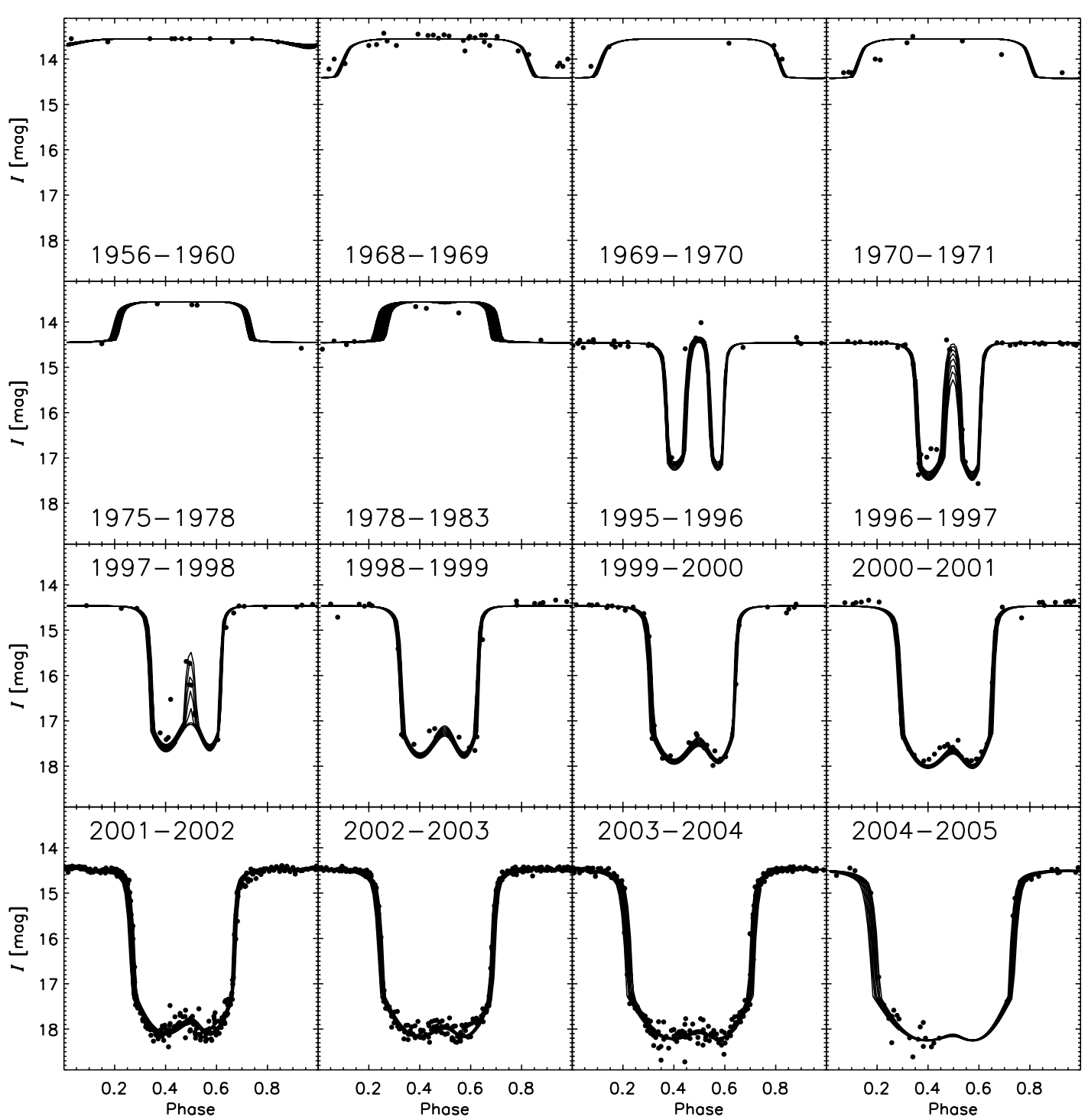

FIG. 12.-Model 3: Calculated and observed light curves. Each panel shows the phased light curve from a particular time range, as observed (solid symbols) and calculated (dots). The boundaries of the time ranges are marked with dashed lines in Fig. 2.

eclipses. Finally, there is one data point in 1997-1998 that is very poorly described: at $I=16.5$ it would seem that the photosphere of star B was partly exposed, but in the model it was behind the screen.

\section{SUMMARY AND DISCUSSION}

Based on the preceding results, we draw two conclusions. First, the newly available data corroborates the theory that $\mathrm{KH}$ $15 \mathrm{D}$ is an eccentric binary system being occulted by the edge of a precessing circumbinary disk. As shown in $\S 5$, the model presented in Paper I succeeds as a quantitative description of $\mathrm{KH}$ $15 \mathrm{D}$, even when confronted with far more data than were available when the model was invented. The best-fitting parameter values are realistic and conform to theoretical expectations. A problem that was overlooked in Paper I - the seemingly unphysical mass ratio of the stars - is fixed with a minor and physically motivated elaboration to the model, namely, the allowance for a more realistic trajectory for the occulting edge across the line of sight. Second, the occultation light curves are well fitted by a model in which each star is surrounded by a more extended halo. This model succinctly accounts for both the photometric variations observed during individual events and the gradual deep- ening of the eclipses. The halo around each star is not symmetric about the center of the star, but in each direction it has a typical scale length of a few stellar radii, and it is brighter in the direction facing the occulting edge. Only the one-dimensional profile of the halo has been measured.

What are these halos? The three broad categories of possible physical interpretations of each halo are (1) luminous material, such as accretion columns or a hot corona, (2) partially extinguished starlight, (3) scattered starlight. The evidence does not permit an unambiguous interpretation, but it does strongly suggest that the halo light has a scattered component. Relative to the uneclipsed light, the mideclipse light has a larger fractional polarization (Agol et al. 2004) and is slightly bluer in color (Hamilton et al. 2001, 2005; Herbst et al. 2002; Knacke et al. 2004; Kusakabe et al. 2005). Enhanced polarization and blueness are hallmarks of scattering by small particles. In contrast, partially extinguished light would be redder than the light source (or the same color, if the particles causing the extinction were large enough). Likewise, the observation of only a small color change argues against self-luminous material in an accretion flow or a hot corona, which would not be expected to have nearly the same effective temperature as the photosphere. 


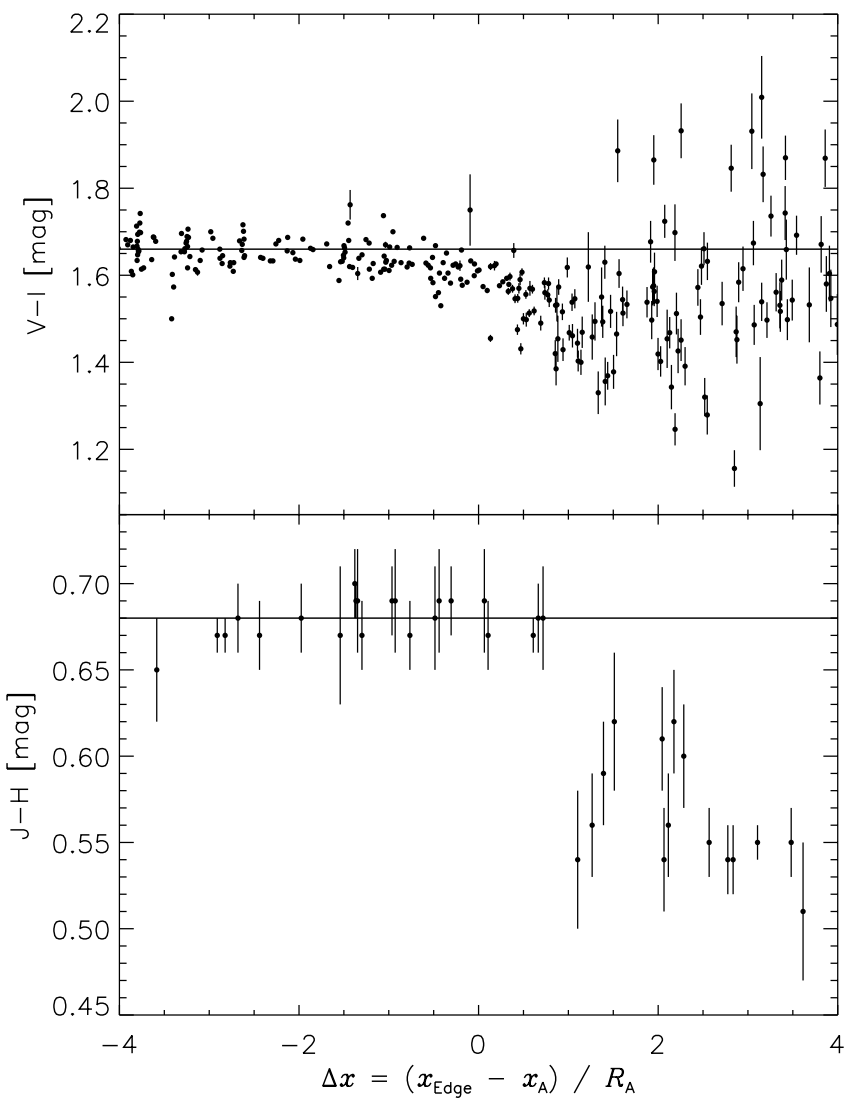

FIG. 13.-Color index of KH 15D, as a function of the distance between the occulting edge and the center of star A. The median color out-of-eclipse is indicated with a solid line. To make this plot, the time stamps on the $V-I_{\mathrm{J}}$ photometry of Hamilton et al. (2005) and the $J-H$ photometry of Kusakabe et al. (2005) were converted into $\left(x_{E}-x_{\mathrm{A}}\right)$, using the model presented in $\S 6$. The halo is seen to be bluer than the photosphere at both optical and near-infrared wavelengths.

Just as the brightness distribution of the halo was determined from $I$-band photometry in $\S 6$, the color distribution of the halo can be measured via multiband photometric monitoring. A first attempt at this is shown in Figure 13, which is analogous to Figure 10, but uses the multiband photometry of Hamilton et al. (2005) and Kusakabe et al. (2005) rather than monochromatic photometry. Higher precision monitoring in multiple bands throughout an ingress or egress will help to resolve the color structure of the halo.

As for the location of the scattering material, the two basic possibilities are the immediate vicinity of each star and the circumbinary disk. Examples of the first scenario are scattering from a corona of hot electrons or from infalling material. A difficulty with this scenario is the near repeatability of the occultation light curve. A circumstellar halo would need to maintain a fixed orientation in space with respect to the occulting feature, despite the rotation of the star and its tidal interaction with its binary companion. Disk scattering, on the other hand, would need to be dominated by forward or back scattering (or some combination of the two) in order to give the appearance of a localized halo around the star. The more general illumination of the disk would not depend strongly on the positions of the stars, because the disk radius of $\sim 3 \mathrm{AU}$ is much larger than the binary semimajor axis of $\sim 0.25$ AU. It is possible, however, that this is a false dichotomy. The disk may extend inward continuously to much smaller distances, and indeed the strong $\mathrm{H} \alpha$ emission, indicative of ongoing accretion, can be taken as evidence for this.
One way in which these possibilities might be distinguished is through the difference in the typical scattering angle. In the case of circumstellar scattering, the received light is scattered over a wide range of angles, as opposed to the forward or back scattering from the disk. The chromatic and polarization signals associated with scattering depend on the typical scattering angle, as well as the size and composition of the particles. Hence, measurements of the polarized light curve, combined with models of the expected polarization signal under various circumstances, may help to determine the size, composition, and spatial distribution of the scattering particles. It would also be possible to determine the velocity of the scattering material relative to the stars through observations of Doppler shifts in the stellar absorption features of the scattered light.

Another interesting feature of the model is the extreme sharpness of the occulting edge. The best evidence for a sharp edge is the slope discontinuity in the light curve that was pointed out in $\S 6$. This "kink" is most easily understood as the point where the sharp occulting edge contacts the rim of the photosphere of the star. If the edge were not sharp, then the time of photospheric contact would be smeared out. The presence of the kink requires that the optical depth of the edge increases from nearly zero to $>3.5$ over a distance that is smaller than a stellar radius. It can also be argued that the edge is sharp on the scale of a stellar radius based on the dramatic changes in the $\mathrm{H} \alpha$ emission and absorption profile that Hamilton et al. (2003) observed during an occultation. The implication is that the occulting edge spatially resolves the $\mathrm{H} \alpha$-emitting region, which is thought to arise within a few stellar radii (i.e., within the magnetosphere).

An independent way to assess the sharpness of the edge would be to measure the Rossiter-McLaughlin effect. In $\S 3$ we predicted the amplitude of this effect for a perfectly sharp and straight edge. In the other extreme, in which the scale length of the edge is much larger than the stellar radius, there is no Rossiter effect. Thus, a sequence of optical spectra with a high signal-to-noise ratio throughout an occultation could be used to determine whether or not the occulting edge resolves the stellar photosphere. If the occulting edge is truly sharp on the scale of the stellar radius, then it can be used to map out the environment of star A on scales unobservable by any other means, just as lunar occultations were used to identify radio and X-ray sources before the necessary angular resolution was available. Spectroscopic monitoring would resolve the velocity structure of the surrounding material. The projected orbital velocity of star A relative to the edge is approximately one stellar radius per day. Thus, a time sampling of $1 \mathrm{hr}$ corresponds to a spatial resolution of $\sim 0.05$ stellar radii, or an angular resolution of $\sim 0.5 \mu$ as at a distance of $760 \mathrm{pc}$. However, the scattering geometry will need to be understood first, to allow the information in the halos to be decoded.

An interesting theoretical question is why the edge is sharp. With a radius of $a_{r}=3 \mathrm{AU}$ and a vertical scale height $h<R_{\odot}$, the circumbinary disk would have $h / a_{r}<10^{-3}$. As pointed out by Chiang \& Murray-Clay (2004), this is at least an order of magnitude smaller than the values generally assumed for T Tauri stars, which are based on the consideration of hydrostatic equilibrium between gas pressure in the disk and the vertical gravitational field provided by the star (see, e.g., Chiang \& Goldreich 1997). One possible resolution is that there is little or no gas over the range of orbital distances that form the occulting feature. Another possibility is that $a_{r}$ is smaller than the dynamical arguments suggest. A third and most intriguing possibility is that the obscuring particles in the disk have settled to the midplane and formed a much sharper layer than the distribution of gas. The dust-settling process has long been investigated as a possible mechanism for 
generating planetesimals, by enhancing the surface density of solid particles until a gravitational instability ensues (Safronov 1969; Goldreich \& Ward 1973; Weidenschilling 1980).

Finally, we end this discussion on a note of urgency. A problem with all of the observations proposed above is that the edge is continuing to advance and will eventually cover star $\mathrm{A}$ at all orbital phases. According to the model of $\S 6$, the photosphere of star A will set for the last time near the beginning of 2008, although that prediction hinges on our extrapolation of the trajectory of the occulting screen. The halo of star A will be visible for several years after the photosphere is hidden. We encourage continued monitoring and will gladly provide predictions of future occultations to interested parties.

We are grateful to V. Grinin and O. Yu. Barsunova for providing their optical data, and to M. Tamura for providing his group's near-infrared data. We thank J. Barranco, E. Ford, S. Gaudi, S. Kenyon, R. Narayan, and G. Rybicki for helpful discussions. S. Gaudi, V. Grinin, and the anonymous referee provided constructive criticism of the manuscript.
Agol, E., Barth, A. J., Wolf, S., \& Charbonneau, D. 2004, ApJ, 600, 781

Badalian, H. S., \& Erastova, L. K. 1970, Astron. Tsirk., 591, 4

Barge, P., \& Viton, M. 2003, ApJ, 593, L117

Barrado y Navascués, D., Mohanty, S., \& Jayawardhana, R. 2004, ApJ, 604, 284

Barsunova, O. Yu., Grinin, V. P., \& Sergeev, S. G. 2005, Astrophys., 48, 1

Bertout, C. 1989, ARA\&A, 27, 351

Bundy, K. A., \& Marcy, G. W. 2000, PASP, 112, 1421

Chiang, E. I., \& Goldreich, P. 1997, ApJ, 490, 368

Chiang, E. I., \& Murray-Clay, R. A. 2004, ApJ, 607, 913

Claret, A. 1998, A\&A, 335, 647 2000, A\&A, 363, 1081

D’Antona, F., \& Mazzitelli, I. 1997, Mem. Soc. Astron. Italiana, 68, 807

Deming, D., Charbonneau, D., \& Harrington, J. 2004, ApJ, 601, L87

Flaccomio, E., Micela, G., Sciortino, S., Favata, F., Corbally, C., \& Tomaney, A. 1999, A\&A, 345, 521

Goldreich, P., \& Ward, W. R. 1973, ApJ, 183, 1051

Grinin, V. P., \& Tambovtseva, L. V. 2002, Astron. Lett., 28, 601

Hamilton, C. M., Herbst, W., Mundt, R., Bailer-Jones, C. A. L., \& Johns-Krull, C. M. 2003, ApJ, 591, L45

Hamilton, C. M., Herbst, W., Shih, C., \& Ferro, A. J. 2001, ApJ, 554, L201

Hamilton, C. M., et al. 2005, AJ, 130, 1896

Herbst, W., Herbst, D. K., Grossman, E. J., \& Weinstein, D. 1994, AJ, 108, 1906

Herbst, W., \& Moran, E. C. 2005, ApJ, 630, 400

Herbst, W., et al. 2002, PASP, 114, 1167

Hillenbrand, L. A., \& White, R. J. 2004, ApJ, 604, 741

Hut, P. 1981, A\&A, 99, 126

Johnson, J. A., Marcy, G. W., Hamilton, C. M., Herbst, W., \& Johns-Krull, C. M. 2004, AJ, 128, 1265

\section{REFERENCES}

Johnson, J. A., \& Winn, J. N. 2004, AJ, 127, 2344

Johnson, J. A., et al. 2005, AJ, 129, 1978

Kearns, K. E., \& Herbst, W. H. 1998, AJ, 116, 261

Klahr, H., \& Bodenheimer, P. 2003, AAS DPS Meeting, 35, 2501

Knacke, R., Fajardo-Acosta, S., \& Tokunaga, A. T. 2004, AJ, 128, 2977

Kusakabe, N., et al. 2005, ApJ, 632, L139

Maffei, P., Ciprini, S., \& Tosti, G. 2005, MNRAS, 357, 1059

McLaughlin, D. B. 1924, ApJ, 60, 22

Press, W. H., Teukolsky, S. A., Vetterling, W. T., \& Flannery, B. P. 1992, Numerical Recipes in C (Cambridge: Cambridge Univ. Press)

Queloz, D., Eggenberger, A., Mayor, M., Perrier, C., Beuzit, J. L., Naef, D., Sivan, J. P., \& Udry, S. 2000, A\&A, 359, L13

Rauch, T., \& Werner, K. 2003, A\&A, 400, 271

Rossiter, R. A. 1924, ApJ, 60, 15

Safronov, V. S. 1969, Evolution of the Protoplanetary Cloud and Formation of the Earth and the Planets (NASA TTF-677; Moscow: Nauka)

Snellen, I. A. G. 2004, MNRAS, 353, L1

Soderblom, D. R., King, J. R., Siess, L., Jones, B. F., \& Fischer, D. 1999, AJ, 118,1301

Sung, H., Bessell, M. S., \& Lee, S.-W. 1997, AJ, 114, 2644

Tokunaga, A. T., et al. 2004, ApJ, 601, L91

van Hamme, W. 1993, AJ, 106, 2096

Walker, M. F. 1956, ApJS, 2, 365

Weidenschilling, S. J. 1980, Icarus, 44, 172

Winn, J. N., Garnavich, P. M., Stanek, K. Z., \& Sasselov, D. D. 2003, ApJ, 593, L121

Winn, J. N., Holman, M. J., Johnson, J. A., Stanek, K. Z., \& Garnavich, P. M. 2004, ApJ, 603, L45

Winn, J. N., et al. 2005, ApJ, 631, 1215

Worek, T. F. 1996, PASP, 108, 962 